Sharif University of Technology
Scientia Iranica
Transactions E: Industrial Engineering
wCIENTIA

\title{
Family of harmonic aggregation operators under intuitionistic fuzzy environment
}

\author{
S. Das* and D. Guha \\ Department of Mathematics, IIT Patna, India.
}

Received 6 October 2015; received in revised form 16 September 2016; accepted 10 October 2016

\section{KEYWORDS}

Intuitionistic fuzzy number;

Aggregation operator;

Harmonic mean;

Ranking;

Multi-attribute group

decision making.

\begin{abstract}
In the process of decision making, the necessity of aggregation of input arguments into a single output becomes a key step; as a result, the selection of an appropriate aggregation operator is a vital aspect. The aim of this contribution is threefold. First, we study algebraic operations of Trapezoidal Intuitionistic Fuzzy Numbers (TrIFNs), and then on the basis of these operational laws, we define four types of harmonic mean operators with TrIFNs. Second, the required properties of the proposed operators are reviewed. After that, an approach based on the proposed operators is introduced to solve a group decision making problem. Finally, a practical example is furnished to demonstrate the applicability of the proposed operators in the decision making context. The contribution ends by introducing comparative analysis of the obtained results.
\end{abstract}

(C) 2017 Sharif University of Technology. All rights reserved.

\section{Introduction}

Aggregation operators have a structural feature that combines a finite number of data points with a single value. Aggregation operators are important tools of information fusion in the decision making problem [13], neural networks [4], fuzzy logic controller [5], and many other fields. Depending on the different nature of information and their relations, various types of aggregation operators have been developed. Among several aggregation operators, the Arithmetic Mean (AM), the Geometric Mean (GM), and the Harmonic Mean (HM) operators are fundamental operators. Based on these basic operators, different types of operators are developed and extended to several environments. Yager [6] proposed the Ordered Weighted Averaging (OWA) operator where the input arguments are organized in order. The Ordered Weighted Geometric

*. Corresponding author.

E-mail address: satyajitnit.das@gmail.com (S.Das)

doi: $10.24200 /$ sci. 2017.4400
(OWG) operator [7] is established based on OWA and GM operators, and the Ordered Weighted Harmonic (OWH) operator [8] is developed by combining OWA and HM operators.

However, it is not always possible that input data are numerical values (crisp values). There may arise some situations where the input data are in imprecise forms rather than numerical values, and this occurs due to lack of information, limitation of knowledge, subjectivity of the human judgment, etc. The imprecise information may be suitably expressed in the form of linguistic variable [9], fuzzy set [10], Intuitionistic Fuzzy Set (IFS) [11] or hesitant fuzzy set [12] which seem to suitably describe an ill-known quantity. In 1965, Zadeh [10] proposed the notion of fuzzy set which captures the imprecise information by assigning a membership degree to each element of the universe. Under the fuzzy environment, a lot of aggregation operators are developed. As an illustration, Fuzzy OWA (FOWA) operator was introduced by Wang and Fan [13]. Different types of harmonic mean operators under fuzzy environment, e.g. fuzzy weighted harmonic mean operator, fuzzy OWH operator, and 
fuzzy hybrid harmonic operators, were developed by $\mathrm{Xu}[14]$. To find the correlation among the arguments, various types of operators, such as power average [15], Choquet integral [16], Bonferroni mean [17] operators, are developed. In the linguistic fuzzy environment, Tian et al. [1] defined linguistic fuzzy weighted fuzzy Bonferroni mean operator and $\mathrm{Yu}$ et al. [12] defined linguistic hesitant fuzzy Heronian mean operator. Ma et al. [3] introduced 2 tuple linguistic aggregation operators on the basis of subjective sensation and objective numerical scales.

By considering the non-membership degree to the fuzzy set, Atanassov [11] proposed IFS theory where the membership degree and non-membership degree do not always complement each other. There may arise some hesitations. Thus, in some cases, IFS theory is more appropriate to deal with inexact information present in real-world applications than the ordinary fuzzy set. Eventually, in less than three decades since its first appearance, IFS theory has been investigated by many authors [18-24] and used for decision making [18,23,25], mathematical programming [20,24,26-28], medical diagnosis [19], to name a few. With this viewpoint, the extension of all aforementioned aggregation operators to intuitionistic fuzzy environment has received considerable attention of many researchers $[29,30]$.

The notion of Intuitionistic Fuzzy Number (IFN) [31-37], defined on the continuous universe, is improved to quantify imprecise quantity. Basically, the concept of IFNs can be viewed as an extensive approach to conventional IFS, which is based on discrete sets. In the information integration process, discrete sets may lose partial information [38], while continuous sets preserve the unity of information and, consequently, are more suitable to model imprecise information rather than former sets. Under this circumstance, extending the concept of discrete sets to continuous sets, IFNs are defined which can more suitably model imprecise data involved in real-world decision making problems [3946]. There are few numbers of aggregation operators existing to aggregate IFNs in the literature. Wang and Zhong [47] proposed the weighted arithmetic and geometric average operators with Trapezoidal IFNs (TrIFNs). Wan and Dong [48] developed Choquet integral operator with Triangular IFNs (TIFNs). With TrIFNs information, in 2016, Wan et al. [2] introduced a fuzzy generalized OWA operator and generalized hybrid weighted averaging operator. In [49], Wan et al. developed arithmetic aggregation operators for triangular Atanassov's IFNs. Furthermore, generalized Choquet integral operator of triangular Atanassov's IFNs was developed by Dong et al. [50]. Wan and Zhu [51] defined triangular intuitionistic fuzzy triple Bonferroni harmonic mean operators. Wu and Cao [52] defined different families of geometric aggregation op- erators to aggregate TrIFNs. Liu and Jin [53] studied Bonferroni mean operator to aggregate trapezoid fuzzy linguistic variables. In 2013, a power-average operator of TrIFNs was introduced by Wan [54] and this operator was used in the decision making problem. In 2015, Wan and Dong [55] developed power geometric operators for TrIFNs. Wan and Yi [56] developed power-average operators for TrIFNs by using strict t-norms and t-conorms. Nowadays, research studies concentrate on interval-valued TrIFNs [57-59] and in this direction, Dong and Wan [57] developed generalized weighted aggregation operators with intervalvalued TrIFNs.

The aim of this contribution is not to cover all the ranges of Multi-Attribute Group Decision Making (MAGDM) problem under intuitionistic fuzzy environment, but merely to discuss the aggregation step. The HM operator is an appropriate tool in the situations, while outliers exist in the data set. If the population consists of few outliers which are much larger than the remaining data, then the HM is the best average operator to use because the HM gives less importance to high-value outliers. For this reason, in this work, we introduce a new aggregation operator, Trapezoidal Intuitionistic Fuzzy Harmonic Mean (TrIFHM) for aggregating TrIFNs. Furthermore, different families of harmonic mean operators are also developed. Particularly, this study focuses on the development of the weighted $\mathrm{HM}$ operator, $\mathrm{OWH}$ mean, induced OWH mean, and hybrid HM operators with TrIFNs information. The main feature of the proposed operators is that the aggregated value is also TrIFN. Then, an approach to MAGDM problem with TrIFNs is developed based on the proposed operators.

The rest of the paper is structured as follows: In Section 2, some definitions and operations related to TrIFNs and a ranking process of TrIFNs are described. Different families of harmonic mean operators are developed in Section 3 and also their properties are studied in this section. In Section 4, an application of the proposed operators in MAGDM problem under intuitionistic fuzzy environment is discussed. In Section 5, a practical example is illustrated. A comparison analysis is also conducted in this section. A concrete conclusion is drawn in Section 6.

\section{Preliminaries}

\subsection{Definition and operations of TrIFNs}

Here, we present a brief introduction to TrIFNs, including their operations, which will be required for our subsequent developments. We start by recalling the definition of TrIFN.

Definition 1. Let $\widetilde{A}=\left[(a, b, c, d), w_{\widetilde{A}}, u_{\widetilde{A}}\right]$ be a TrIFN defined on real-line $\mathbb{R}$. The membership and 
non-membership functions of $\widetilde{A}$ are respectively given as follows:

$$
\mu_{\widetilde{A}}(x)= \begin{cases}\frac{(x-a) w_{\widetilde{A}}}{(b-a)}, & \text { for } a \leq x<b, \\ w_{\widetilde{A}}, & \text { for } b \leq x \leq c, \\ \frac{(d-x) w_{\widetilde{A}}}{(d-c)}, & \text { for } c<x \leq d, \\ 0, & \text { for } x<a, x>d .\end{cases}
$$

and:

$$
\nu_{\widetilde{A}}(x)= \begin{cases}\frac{(b-x)+(x-a) u_{\tilde{A}}}{(b-a)}, & \text { for } a \leq x<b, \\ u_{\widetilde{A}}, & \text { for } b \leq x \leq c \\ \frac{(x-c)+(d-x) u_{\tilde{A}}}{(d-c)}, & \text { for } c<x \leq d, \\ 1, & \text { for } x<a, x>d,\end{cases}
$$

where $w_{\widetilde{A}}$ represents the maximum membership degree, whereas $u_{\widetilde{A}}$ is the minimum degree of non-membership of element $x \in \widetilde{A}$. They follow the relation: $0 \leq$ $w_{\widetilde{A}}, u_{\widetilde{A}} \leq 1 ; 0 \leq w_{\widetilde{A}}+u_{\widetilde{A}} \leq 1$. Function $\pi_{\widetilde{A}}(x)=$ $1-\mu_{\widetilde{A}}(x)-\nu_{\widetilde{A}}(x)$ is called hesitancy of element $x$ in $\widetilde{A}$. However, if $b=c$, then TrIFN is reduced to a TIFN. In the context of decision making problem, TrIFN $\tilde{A}$ allows one to simulate human cognitive processes in a more appropriate manner than the traditional fuzzy number. The interpretation of TrIFNs is found in [31].

Definition 2 [55]. If $a \geq 0$ and one of $a, b, c$, and $d$ is nonzero, then $\operatorname{TrIFN} \widetilde{A}=\left[(a, b, c, d) ; w_{\widetilde{A}}, u_{\widetilde{A}}\right]$ is called positive TrIFN.

Definition 3. Two TrIFNs $\widetilde{A}=\left[\left(a_{1}, b_{1}, c_{1}, d_{1}\right) ; w_{\widetilde{A}}\right.$, $\left.u_{\widetilde{A}}\right]$ and $\widetilde{B}=\left[\left(a_{2}, b_{2}, c_{2}, d_{2}\right) ; w_{\widetilde{B}}, u_{\widetilde{B}}\right]$ are said to be equal if $a_{1}=a_{2}, b_{1}=b_{2}, c_{1}=c_{2}, d_{1}=d_{2}, w_{\widetilde{A}}=w_{\widetilde{B}}$ and $u_{\widetilde{A}}=u_{\widetilde{B}}$.

Definition 4 [52]. Let $\widetilde{A}=\left[\left(a_{1}, b_{1}, c_{1}, d_{1}\right) ; w_{\widetilde{A}}, u_{\widetilde{A}}\right]$ and $\widetilde{B}=\left[\left(a_{2}, b_{2}, c_{2}, d_{2}\right) ; w_{\widetilde{B}}, u_{\widetilde{B}}\right]$ be two TrIFNs and $\alpha \geq 0$ be a scalar. Then:

- $\widetilde{A}+\widetilde{B}=\left[\left(a_{1}+a_{2}, b_{1}+b_{2}, c_{1}+c_{2}, d_{1}+d_{2}\right) ; w_{\widetilde{A}}+\right.$ $\left.w_{\widetilde{B}}-w_{\widetilde{A}} w_{\widetilde{B}}, u_{\widetilde{A}} u_{\widetilde{B}}\right] ;$

- $\widetilde{A} \times \widetilde{B}=\left[\left(a_{1} a_{2}, b_{1} b_{2}, c_{1} c_{2}, d_{1} d_{2}\right) ; w_{\widetilde{A}} w_{\widetilde{B}}, u_{\widetilde{A}}+u_{\widetilde{B}}-\right.$ $\left.u_{\widetilde{A}} u_{\widetilde{B}}\right]$;

- $\alpha \widetilde{A}=\left[\left(\alpha a_{1}, \alpha b_{1}, \alpha c_{1}, \alpha d_{1}\right) ; 1-\left(1-w_{\widetilde{A}}\right)^{\alpha}, u_{\widetilde{A}}^{\alpha}\right]$;

- $\widetilde{A}^{\alpha}=\left[\left(a_{1}^{\alpha}, b_{1}^{\alpha}, c_{1}^{\alpha}, d_{1}^{\alpha}\right) ; w_{\widetilde{A}}^{\alpha}, 1-\left(1-u_{\widetilde{A}}\right)^{\alpha}\right]$.

Definition 5. Let $\widetilde{A}_{1}=\left[\left(a_{1}, b_{1}, c_{1}, d_{1}\right) ; w_{\widetilde{A}_{1}}, u_{\widetilde{A}_{1}}\right]$ be a positive TrIFN, then:

$$
\frac{1}{\widetilde{A}_{1}}=\widetilde{A}_{1}^{-1}=\left[\left(\frac{1}{d_{1}}, \frac{1}{c_{1}}, \frac{1}{b_{1}}, \frac{1}{a_{1}}\right) ; w_{\widetilde{A}_{1}}, u_{\widetilde{A}_{1}}\right] .
$$

\subsection{Ranking process of TrIFNs}

To solve a MAGDM problem, ranking of alternatives is one of the key steps. In comparing IFNs, researchers have introduced various ranking methods. One of such methods that is employed in the present study is a centroid-based ranking method of TrIFNs [60]. Now, we present the method to compute the centroid point of a TrIFN by using the following steps.

- Step I: Computation of $X$ coordinate. Let $\widetilde{A}=$ $\left[(a, b, c, d) ; w_{\widetilde{A}}, u_{\tilde{A}}\right]$ be a TrIFN whose membership and non-membership functions are defined in Eqs. (1) and (2), respectively, and depicted in Figure 1. Let $f_{\widetilde{A}}^{L}:[a, b] \rightarrow\left[0, w_{\tilde{A}}\right], f_{\widetilde{A}}^{R}:[c, d] \rightarrow$ $\left[0, w_{\tilde{A}}\right]$ be the left and right parts of membership function $\mu_{\widetilde{A}}$, respectively, and $g_{\widetilde{A}}^{L}:[a, b] \rightarrow\left[0, u_{\widetilde{A}}\right]$, $g_{\widetilde{A}}^{R}:[c, d] \rightarrow\left[0, u_{\widetilde{A}}\right]$ be left and right parts of nonmembership function $\nu_{\widetilde{A}}$ of $\operatorname{TrIFN} \widetilde{A}$, respectively. Functions $f_{\widetilde{A}}^{L}(x), f_{\widetilde{A}}^{R}(x), g_{\widetilde{A}}^{L}(x)$, and $g_{\widetilde{A}}^{R}(x)$ can be mathematically represented by using Eqs. (1) and (2) as follows:

$$
\begin{array}{rlrl}
f_{\widetilde{A}}^{L}(x) & =\frac{w_{\widetilde{A}}(x-a)}{b-a}, & \text { for } a \leq x \leq b, \\
g_{\widetilde{A}}^{L}(x)=\frac{(x-b)+u_{\widetilde{A}}(a-x)}{a-b}, & \text { for } a \leq x \leq b, \\
f_{\widetilde{A}}^{R}(x)=\frac{w_{\widetilde{A}}(d-x)}{d-c}, & \text { for } c \leq x \leq d, \\
g_{\widetilde{A}}^{R}(x)=\frac{(x-c)+u_{\widetilde{A}}(d-x)}{d-c}, & \text { for } c \leq x \leq d .
\end{array}
$$

Let $\left(X_{\widetilde{A}}, Y_{\widetilde{A}}\right)$ be the centroid point of $\widetilde{A}$. To compute the centroid point, the area under

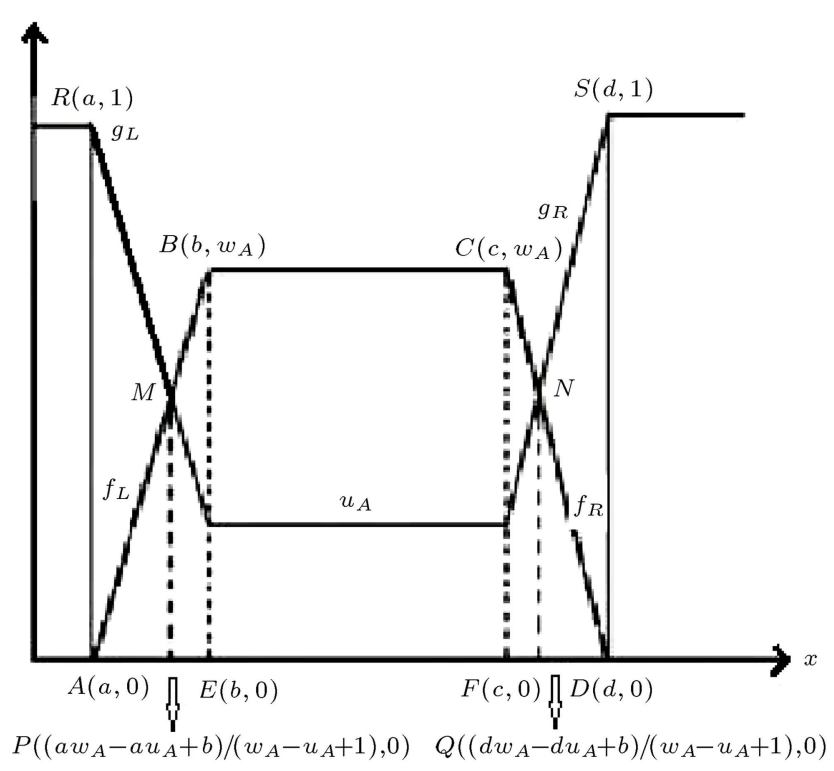

Figure 1. Trapezoidal intuitionistic fuzzy number A. 
both membership and non-membership functions is considered. First, the whole area is partitioned into five rectangles (Figure 1): APMR, PEBM, EFCB, FQNC, and QDSN. The coordinates of the corner points of these rectangles are given as follows:

$$
\begin{aligned}
& A:(a, 0), \quad B:\left(b, w_{\tilde{A}}\right), \quad C:\left(c, w_{\tilde{A}}\right), \\
& D:(d, 0), \quad E:(b, 0), \quad F:(c, 0), \\
& S:(d, 1), \quad P:\left(\frac{a w_{\widetilde{A}}-a u_{\widetilde{A}}+b}{w_{\widetilde{A}}-u_{\widetilde{A}}+1}, 0\right), \\
& Q:\left(\frac{d w_{\widetilde{A}}-d u_{\widetilde{A}}+c}{w_{\widetilde{A}}-u_{\widetilde{A}}+1}, 0\right), \quad R:(a, 1), \\
& M:\left(\frac{a w_{\tilde{A}}-a u_{\tilde{A}}+b}{w_{\widetilde{A}}-u_{\widetilde{A}}+1}, \frac{w_{\tilde{A}}}{w_{\tilde{A}}-u_{\tilde{A}}+1}\right), \\
& N:\left(\frac{d w_{\widetilde{A}}-d u_{\widetilde{A}}+c}{w_{\widetilde{A}}-u_{\widetilde{A}}+1}, \frac{w_{\widetilde{A}}}{w_{\widetilde{A}}-u_{\widetilde{A}}+1}\right) .
\end{aligned}
$$

Then, $X$ coordinate $\left(X_{\tilde{A}}\right)$ of the centroid point of TrIFN $\widetilde{A}$ can be computed as follows:

$$
X_{\widetilde{A}}=\frac{\int_{a}^{P_{x}} x g_{\widetilde{A}}^{L} d x+\int_{P_{x}}^{b} x f_{\widetilde{A}}^{L} d x+\int_{b}^{c} x w d x+\int_{c}^{Q_{x}} x f_{\widetilde{A}}^{R} d x+\int_{Q_{x}}^{d} x g_{\widetilde{A}}^{R} d x}{\int_{a}^{P_{x}} g_{\widetilde{A}}^{L} d x+\int_{P_{x}}^{b} f_{\widetilde{A}}^{L} d x+\int_{b}^{c} w d x+\int_{c}^{Q_{x}} f_{\widetilde{A}}^{R} d x+\int_{Q_{x}}^{d} g_{\widetilde{A}}^{R} d x},
$$

where $P_{x}$ and $Q_{x}$ are $X$ coordinates of points $P$ and $Q$, respectively.

- Step II: Computation of $Y$ coordinate. In order to compute $Y$ coordinate of centroid point, the inverse functions of left and right parts of the membership and non-membership functions of TrIFN $\widetilde{A}$ are considered. The whole area is partitioned into three bounded areas (Figure 2): ABCD, DNS, and ARM, where the coordinates of the corner points of these areas are provided in Step I. As $f_{\widetilde{A}}^{L}, f_{\widetilde{A}}^{R}, g_{\widetilde{A}}^{L}$ and $g_{\widetilde{A}}^{R}$ are strictly monotonic and continuous functions, consequently their inverse functions should exist also be strictly monotonic and continuous. Let $h_{\widetilde{A}}^{L}$ : $[0, w] \rightarrow[a, b]$ and $h_{\widetilde{A}}^{R}:[0, w] \rightarrow[c, d]$ be the inverse functions of $f_{\widetilde{A}}^{L}$ and $f_{\widetilde{A}}^{R}$, respectively. Let $g_{\widetilde{A}}^{L}$ inverse

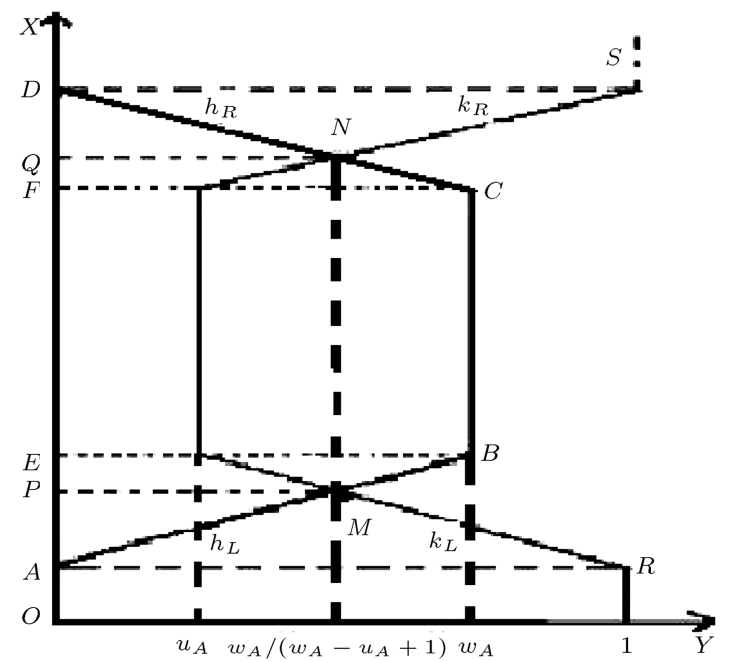

Figure 2. Inverse of trapezoidal intuitionistic fuzzy number A.

functions of $g_{\widetilde{A}}^{L}$ and $g_{\widetilde{A}}^{R}$ be $k_{\widetilde{A}}^{L}[0, u] \rightarrow[a, b]$ and $k_{\widetilde{A}}^{R}$ : $[0, u] \rightarrow[c, d]$, respectively. Functions $h_{\widetilde{A}}^{L}(y), h_{\widetilde{A}}^{R}(y)$, $k_{\widetilde{A}}^{L}(y)$ and $k_{\widetilde{A}}^{R}(y)$ can be mathematically represented as follows:

$$
\begin{array}{ll}
h_{\widetilde{A}}^{L}(y)=a+\frac{(b-a) y}{w}, & \text { for } 0 \leq y \leq w, \\
k_{\widetilde{A}}^{L}(y)=\frac{(a-b) y+(b-a u)}{1-u}, & \text { for } u \leq y \leq 1, \\
h_{\widetilde{A}}^{R}(y)=d-\frac{(d-c) y}{w}, & \text { for } 0 \leq y \leq w, \\
k_{\widetilde{A}}^{R}(y)=\frac{(d-c) y+(c-d u)}{1-u}, & \text { for } u \leq y \leq 1 .
\end{array}
$$

Then, $Y$ coordinate $\left(Y_{\widetilde{A}}\right)$ of the centroid point of TrIFN $\widetilde{A}$ can be computed as shown in Box I, where $M_{y}$ and $N_{y}$ are $Y$ coordinates of points $M$ and $N$, respectively.

The ranking of two TrIFNs $\widetilde{A}$ and $\widetilde{B}$ with centroid points $\left(X_{\widetilde{A}}, Y_{\widetilde{A}}\right)$ and $\left(X_{\widetilde{B}}, Y_{\widetilde{B}}\right)$ can be done in the following way:
(a) If $X_{\widetilde{A}}<X_{\widetilde{B}}$, then $\widetilde{A}<\widetilde{B}$;
(b) If $X_{\widetilde{A}}=X_{\widetilde{B}}$, then

$$
\begin{array}{r}
\text { if } Y_{\widetilde{A}}<Y_{\widetilde{B}} \text {, then } \widetilde{A}<\widetilde{B} \text {; } \\
\quad \text { else } Y_{\widetilde{A}}=Y_{\widetilde{B}} \text {, then } \widetilde{A}=\widetilde{B} \text {. }
\end{array}
$$

$$
Y_{\widetilde{A}}=\frac{\int_{0}^{w} y\left(h_{\widetilde{A}}^{R}-h_{\widetilde{A}}^{L}\right) d y+\left[\int_{0}^{1} y d \cdot d y-\int_{0}^{N_{y}} y h_{\widetilde{A}}^{R} d y-\int_{N_{y}}^{1} y k_{\widetilde{A}}^{R} d y\right]+\left[\int_{0}^{M_{y}} y h_{\widetilde{A}}^{L} d y+\int_{M_{y}}^{1} y k_{\widetilde{A}}^{L} d y-\int_{0}^{1} a y d y\right]}{\int_{0}^{w}\left(h_{\widetilde{A}}^{R}-h_{\widetilde{A}}^{L}\right) d y+\left[\int_{0}^{1} d . d y-\int_{0}^{N_{y}} h_{\widetilde{A}}^{R} d y-\int_{N_{y}}^{1} k_{\widetilde{A}}^{R} d y\right]+\left[\int_{0}^{M_{y}} h_{\widetilde{A}}^{L} d y+\int_{M_{y}}^{1} k_{\widetilde{A}}^{L} d y-\int_{0}^{1} a d y\right]} .
$$




\section{Intuitionistic harmonic mean operator}

In this section, HM operator for crisp numbers is discussed first, and then this operator is developed under intuitionistic fuzzy environment.

\subsection{Harmonic mean}

The HM and weighted HM operators can be defined as follows.

Definition 6. Let $\check{a}_{1}, \check{a}_{2}, \cdots, \check{a}_{n}$ be $n$ real numbers. Then, HM operator is defined as follows:

$$
H\left(\check{a}_{1}, \check{a}_{2}, \cdots, \check{a}_{n}\right)=\frac{n}{\sum_{j=1}^{n} \frac{1}{\check{a}_{j}}},
$$

that is, HM is the reciprocal of the AM of the reciprocals.

Definition 7. Let $\check{a}_{1}, \check{a}_{2}, \cdots, \check{a}_{n}$ be $n$ real numbers. Then, weighted HM operator is defined by:

$$
H_{\tau}\left(\check{a}_{1}, \check{a}_{2}, \cdots, \check{a}_{n}\right)=\frac{1}{\sum_{j}^{n} \frac{\tau_{j}}{\check{a}_{j}}},
$$

where $\tau=\left(\tau_{1}, \tau_{2}, \cdots, \tau_{n}\right)^{T}$ is a weight vector of $\check{a}_{j}(j=$ $1,2, \cdots, n), \tau_{j} \in[0,1]$ and $\sum_{j=1}^{n} \tau_{j}=1$.

When outliers exist in the sample data set, HM operator would be the best choice [61] to aggregate the data as the HM gives less importance to high-value outliers.

For example, suppose that there are thirteen bars (Figure 3) of almost similar height in a sample and there is one bar which is more than three times longer. Consequently, this outlier may strongly affect the AM and may provide an average value which is biased due to the presence of this bar. However, HM provides the less biased result as it gives less importance to outliers. In this example, the aggregated value obtained by AM is approximately 47.7 , while it is approximately 41 by $\mathrm{HM}$, i.e., in this case, the AM overstates the average value by over $15 \%$. Hence, if the aim is to compute the average value of data where few outliers exist, then HM is the best one.

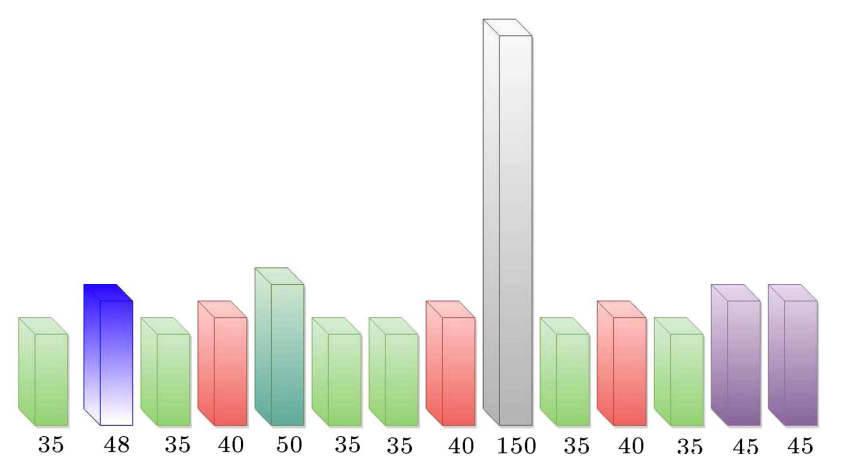

Figure 3. Bar diagram of thirteen data with one outlier.

\subsection{Family of harmonic mean operators of $\operatorname{TrIFNs}$}

The aforementioned discussion motivates us to develop harmonic mean operator in the intuitionistic fuzzy environment. The description of this operator is given below. Let $\mho$ be the set of positive TrIFNs

Definition 8. Let $\widetilde{A}_{j}=\left[\left(a_{j}, b_{j}, c_{j}, d_{j}\right) ; w_{\widetilde{A}_{j}}, u_{\widetilde{A}_{j}}\right]$ $(j=1,2, . ., n)$ be a collection of positive TrIFNs. A Trapezoidal Intuitionistic Fuzzy Weighted Harmonic Mean (TrIFWHM) operator is a mapping TrIFWHM : $\mho^{n} \rightarrow \mho$ which is defined by:

$$
\operatorname{Tr} \operatorname{IFWHM}\left(\widetilde{A}_{1}, \widetilde{A}_{2}, \cdots, \widetilde{A}_{n}\right)=\frac{1}{\sum_{j=1}^{n} \frac{\tau_{j}}{\widetilde{A}_{j}}}
$$

where $\tau=\left(\tau_{1}, \tau_{2}, \cdots, \tau_{n}\right)^{T}$ is a weight vector of TrIFNs $\widetilde{A}_{j}(j=1,2, \cdots, n), \tau_{j} \in[0,1]$, and $\sum_{j=1}^{n} \tau_{j}=1$. Furthermore, if weight vector $\tau=\left(\tau_{1}, \tau_{2}, \cdots, \tau_{n}\right)^{T}$ reduces to $\tau=\left(\frac{1}{n}, \frac{1}{n}, \cdots, \frac{1}{n}\right)^{T}$, then operator TrIFWHM reduces to Trapezoidal Intuitionistic Fuzzy Harmonic Mean (TrIFHM) operator which is defined below.

Definition 9. Let $\widetilde{A}_{j}(j=1,2, \cdots, n)$ be a collection of positive TrIFNs. A TrIFHM operator is a mapping TrIFHM : $\mho^{n} \rightarrow \mho$ which is defined by:

$$
\operatorname{Tr} \operatorname{IFHM}\left(\widetilde{A}_{1}, \widetilde{A}_{2}, \cdots, \widetilde{A}_{n}\right)=\frac{n}{\sum_{j=1}^{n} \frac{1}{\widetilde{A}_{j}}} .
$$

Theorem 1. Let $\widetilde{A}_{j}(j=1,2, \cdots, n)$ be a collection of positive TrIFNs and $\tau=\left(\tau_{1}, \tau_{2}, \cdots, \tau_{n}\right)^{T}$ be a weight vector of $\widetilde{A}_{j}$ where $\tau_{j} \in[0,1], \sum_{j=1}^{n} \tau_{j}=1$. Then, the aggregation result by using TrIFWHM operator is again a $\operatorname{TrIFN}$ and:

$$
\begin{aligned}
\operatorname{Tr} \operatorname{IF} & \operatorname{HhM}\left(\widetilde{A}_{1}, \widetilde{A}_{2}, \cdots, \widetilde{A}_{n}\right) \\
= & {\left[\left(\frac{1}{\sum_{j=1}^{n} \frac{\tau_{j}}{a_{j}}}, \frac{1}{\sum_{j=1}^{n} \frac{\tau_{j}}{b_{j}}}, \frac{1}{\sum_{j=1}^{n} \frac{\tau_{j}}{c_{j}}}, \frac{1}{\sum_{j=1}^{n} \frac{\tau_{j}}{d_{j}}}\right) ;\right.} \\
& \left.1-\prod_{j}^{n}\left(1-w_{\widetilde{A}_{j}}\right)^{\tau_{j}}, \prod_{j}^{n} u_{\widetilde{A}_{j}}^{\tau_{j}}\right]
\end{aligned}
$$

Proof: Proof of this theorem can be done by the principle of mathematical induction.

When $n=2$, then $\operatorname{TrIFWPHM}\left(\tilde{A}_{1}, \tilde{A}_{2}\right)$ is calculated as shown in Box II. So, Eq. (9) is true for $n=2$.

Suppose that Eq. (9) holds for $n=k$, i.e.:

$$
\operatorname{Tr} \operatorname{IFWHM}\left(\widetilde{A}_{1}, \widetilde{A}_{2}, \cdots, \widetilde{A}_{k}\right)
$$




$$
\begin{aligned}
& \operatorname{TrIFWPHM}\left(\widetilde{A}_{1}, \widetilde{A}_{2}\right)=\frac{1}{\frac{\tau_{1}}{\widetilde{A}_{1}}+\frac{\tau_{2}}{\widetilde{A}_{2}}}=\frac{1}{\left[\left(a_{1}, b_{1}, c_{1}, d_{1}\right) w_{\left.\tilde{A}_{1}, u_{\tilde{A}_{1}}\right]}+\frac{\tau_{1}}{\left[\left(a_{2}, b_{2}, c_{2}, d_{2}\right) ; w_{\tilde{A}_{2}}, u_{\tilde{A}_{2}}\right]}\right.} \\
& =\frac{1}{\tau_{1}\left[\left(\frac{1}{d_{1}}, \frac{1}{c_{1}}, \frac{1}{b_{1}}, \frac{1}{a_{1}}\right) ; w_{1}, u_{1}\right]+\tau_{2}\left[\left(\frac{1}{d_{2}}, \frac{1}{c_{2}}, \frac{1}{b_{2}}, \frac{1}{a_{2}}\right) ; w_{\widetilde{A}_{2}}, u_{\widetilde{A}_{j}}\right]} \\
& =\frac{1}{\left[\left(\frac{\tau_{1}}{d_{1}}, \frac{\tau_{1}}{c_{1}}, \frac{\tau_{1}}{b_{1}}, \frac{\tau_{1}}{a_{1}}\right) ; 1-\left(1-w_{\widetilde{A}_{1}}\right)^{\tau_{1}}, u_{\widetilde{A}_{1}}^{\tau_{1}}\right]+\left[\left(\frac{\tau_{2}}{d_{2}}, \frac{\tau_{2}}{c_{2}}, \frac{\tau_{2}}{b_{2}}, \frac{\tau_{2}}{a_{2}}\right) ; 1-\left(1-w_{\widetilde{A}_{2}}\right)^{\tau_{2}}, u_{\widetilde{A}_{2}}^{\tau_{2}}\right]} \\
& =\frac{1}{\left[\left(\frac{\tau_{1}}{d_{1}}+\frac{\tau_{2}}{d_{2}}, \frac{\tau_{1}}{c_{1}}+\frac{\tau_{2}}{c_{2}}, \frac{\tau_{1}}{b_{1}}+\frac{\tau_{2}}{b_{2}}, \frac{\tau_{1}}{a_{1}}+\frac{\tau_{2}}{a_{2}}\right) ; 1-\left(1-w_{\widetilde{A}_{1}}\right)^{\tau_{1}}+1-\left(1-w_{\widetilde{A}_{2}}\right)^{\tau_{2}}-\left(1-\left(1-w_{\widetilde{A}_{1}}\right)^{\tau_{1}}\right)\left(1-\left(1-w_{\widetilde{A}_{2}}\right)^{\tau_{2}}\right), u_{\widetilde{A}_{1}}^{\tau_{1}} u_{\widetilde{A}_{2}}^{\tau_{2}}\right]} \\
& =\frac{1}{\left[\left(\frac{\tau_{1}}{d_{1}}+\frac{\tau_{2}}{d_{2}}, \frac{\tau_{1}}{c_{1}}+\frac{\tau_{2}}{c_{2}}, \frac{\tau_{1}}{b_{1}}+\frac{\tau_{2}}{b_{2}}, \frac{\tau_{1}}{a_{1}}+\frac{\tau_{2}}{a_{2}}\right) ; 1-\prod_{j=1}^{2}\left(1-w_{\widetilde{A}_{j}}\right)^{\tau_{j}}, \prod_{j=1}^{2} u_{\widetilde{A}_{j}}^{\tau_{j}}\right]} \\
& =\left[\left(\frac{1}{\frac{\tau_{1}}{a_{1}}+\frac{\tau_{2}}{a_{2}}}, \frac{1}{\frac{\tau_{1}}{b_{1}}+\frac{\tau_{2}}{b_{2}}}, \frac{1}{\frac{\tau_{1}}{c_{1}}+\frac{\tau_{2}}{c_{2}}}, \frac{1}{\frac{\tau_{1}}{d_{1}}+\frac{\tau_{2}}{d_{2}}}\right) ; 1-\prod_{j=1}^{2}\left(1-w_{\widetilde{A}_{j}}\right)^{\tau_{j}}, \prod_{j=1}^{2} u_{\widetilde{A}_{j}}^{\tau_{j}}\right]
\end{aligned}
$$

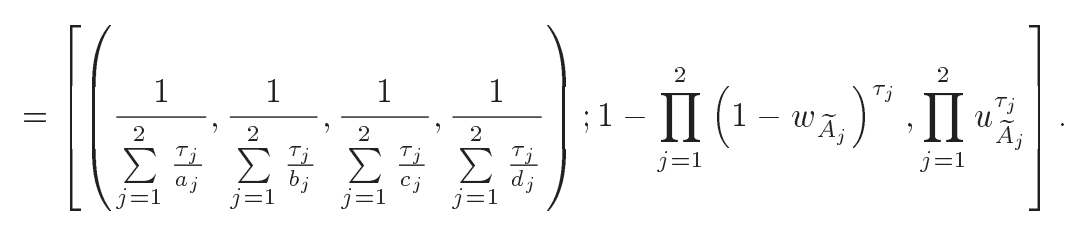

Box II

$$
\begin{aligned}
= & {\left[\left(\frac{1}{\sum_{j=1}^{k} \frac{\tau_{j}}{a_{j}}}, \frac{1}{\sum_{j=1}^{k} \frac{\tau_{j}}{b_{j}}}, \frac{1}{\sum_{j=1}^{k} \frac{\tau_{j}}{c_{j}}}, \frac{1}{\sum_{j=1}^{k} \frac{\tau_{j}}{d_{j}}}\right) ;\right.} \\
& \left.1-\prod_{j=1}^{k}\left(1-w_{\widetilde{A}_{j}}\right)^{\tau_{j}}, \prod_{j=1}^{k} u_{\widetilde{A}_{j}}^{\tau_{j}}\right] .
\end{aligned}
$$

Now for $n=k+1$, using above expression and operational laws, we will have:

$$
\begin{aligned}
\operatorname{Tr} \operatorname{IFWHM}\left(\widetilde{A}_{1}, \widetilde{A}_{2}, \cdots, \widetilde{A}_{k}, \widetilde{A}_{k+1}\right) & =\frac{1}{\sum_{j=1}^{k} \frac{\tau_{j}}{\widetilde{A}_{j}}+\frac{\tau_{k+1}}{\widetilde{A}_{k+1}}} \\
= & {\left[\left(\frac{1}{\sum_{j=1}^{k+1} \frac{\tau_{j}}{a_{j}}}, \frac{1}{\sum_{j=1}^{k+1} \frac{\tau_{j}}{b_{j}}}, \frac{1}{\sum_{j=1}^{k+1} \frac{\tau_{j}}{c_{j}}}, \frac{1}{\sum_{j=1}^{k+1} \frac{\tau_{j}}{d_{j}}}\right) ;\right.} \\
& \left.1-\prod_{j=1}^{k+1}\left(1-w_{\widetilde{A}_{j}}\right)^{\tau_{j}}, \prod_{j=1}^{k+1} u_{\widetilde{A}_{j}}^{\tau_{j}}\right] .
\end{aligned}
$$

So, Eq. (9) is true for $n=k+1$. Hence, by mathematical induction, Eq. (9) holds for all $n$.

Now, we shall study desirable properties of the proposed TrIFWHM operator.

(P1) Idempotency: If all of input arguments $\widetilde{A}_{j}$ are equal and $\widetilde{A}_{j}=\widetilde{A}=\left[(a, b, c, d) ; w_{\widetilde{A}}, u_{\widetilde{A}}\right], \forall j$. Then:

$$
\begin{aligned}
& \operatorname{Tr} \operatorname{IFWhm}\left(\widetilde{A}_{1}, \widetilde{A}_{2}, \cdots, \widetilde{A}_{n}\right) \\
& \quad=\operatorname{Tr} \operatorname{TrWhM}(\widetilde{A}, \widetilde{A}, \cdots, \widetilde{A})=\widetilde{A}
\end{aligned}
$$

Proof: We have

$$
\begin{aligned}
& \operatorname{TrIFWHM}\left(\widetilde{A}_{1}, \widetilde{A}_{2}, \cdots, \widetilde{A}_{n}\right) \\
& =\left[\left(\frac{1}{\sum_{j=1}^{n} \frac{\tau_{j}}{a_{j}}}, \frac{1}{\sum_{j=1}^{n} \frac{\tau_{j}}{b_{j}}}, \frac{1}{\sum_{j=1}^{n} \frac{\tau_{j}}{c_{j}}}, \frac{1}{\sum_{j=1}^{n} \frac{\tau_{j}}{d_{j}}}\right)\right. \\
& \left.1-\prod_{j}^{n}\left(1-w_{\widetilde{A}_{j}}\right)^{\tau_{j}}, \prod_{j}^{n} u_{\widetilde{A}_{j}}^{\tau_{j}}\right]
\end{aligned}
$$




$$
\begin{aligned}
= & {\left[\left(\frac{1}{\frac{\left(\sum_{j=1}^{n} \tau_{j}\right)}{a}, \frac{1}{\left(\sum_{j=1}^{n} \tau_{j}\right)}}, \frac{1}{b} \frac{1}{\left(\sum_{j=1}^{n} \tau_{j}\right)} \frac{1}{c}\right)\right.} \\
& \left.1-\left(1-w_{\widetilde{A}}\right)^{\left.\sum_{j=1}^{n} \tau_{j} \tau_{j}\right)}, u_{\tilde{A}}^{\sum_{\tilde{A}}^{n} \tau_{j}}\right] \\
= & {\left[(a, b, c, d) ; w_{\widetilde{A}}, u_{\widetilde{A}}\right]\left(\because \sum_{j=1}^{n} \tau_{j}=1\right)=\widetilde{A} . }
\end{aligned}
$$

(P2) Monotonicity: Let $\widetilde{A}_{j}=\left[\left(a_{j}, b_{j}, c_{j}, d_{j}\right) ; w_{\widetilde{A}_{j}}\right.$, $\left.u_{\widetilde{A}_{j}}\right]$ and $\overline{\widetilde{A}}_{j}=\left[\left(\bar{a}_{j}, \bar{b}_{j}, \bar{c}_{j}, \bar{d}_{j}\right) ; \bar{w}_{\widetilde{A}_{j}}, \bar{u}_{\widetilde{A}_{j}}\right](j=1,2, \cdots$, $n)$ be two collections of positive TrIFNs. If $a_{j} \leq \bar{a}_{j}$, $b_{j} \leq \bar{b}_{j}, c_{j} \leq \bar{c}_{j}, d_{j} \leq \bar{d}_{j}, w_{\widetilde{A}_{j}} \leq \bar{w}_{\widetilde{A}_{j}}$ and $u_{\widetilde{A}_{j}} \geq \bar{u}_{\widetilde{A}_{j}}$ $\forall j$, then:

$$
\begin{aligned}
& \operatorname{Tr} \operatorname{IFWhM}\left(\widetilde{A}_{1}, \widetilde{A}_{2}, \cdots, \widetilde{A}_{n}\right) \\
& \quad \leq \operatorname{TrIFWHM}\left(\overline{\widetilde{A}}_{1}, \overline{\widetilde{A}}_{2}, \cdots, \overline{\widetilde{A}}_{n}\right) .
\end{aligned}
$$

Proof: $\quad$ Since $a_{j} \leq \bar{a}_{j}$ and $\tau_{j} \geq 0, \forall j$

$$
\therefore \frac{\tau_{j}}{a_{j}} \geq \frac{\tau_{j}}{\bar{a}_{j}} \Rightarrow \sum_{j=1}^{n} \frac{\tau_{j}}{a_{j}} \geq \sum_{j=1}^{n} \frac{\tau_{j}}{\bar{a}_{j}} \Rightarrow \frac{1}{\sum_{j=1}^{n} \frac{\tau_{j}}{a_{j}}} \leq \frac{1}{\sum_{j=1}^{n} \frac{\tau_{j}}{\bar{a}_{j}}} .
$$

Similarly:

$$
\frac{1}{\sum_{j=1}^{n} \frac{\tau_{j}}{b_{j}}} \leq \frac{1}{\sum_{j=1}^{n} \frac{\tau_{j}}{\bar{b}_{j}}}, \quad \frac{1}{\sum_{j=1}^{n} \frac{\tau_{j}}{c_{j}}} \leq \frac{1}{\sum_{j=1}^{n} \frac{\tau_{j}}{\bar{c}_{j}}}
$$

and

$$
\frac{1}{\sum_{j=1}^{n} \frac{\tau_{j}}{d_{j}}} \leq \frac{1}{\sum_{j=1}^{n} \frac{\tau_{j}}{\overline{d_{j}}}}
$$

Since

$$
\begin{aligned}
& w_{\widetilde{A}_{j}} \leq \bar{w}_{\widetilde{A}_{j}} \Rightarrow 1-w_{\widetilde{A}_{j}} \geq 1-\bar{w}_{\widetilde{A}_{j}} \\
& \Rightarrow \prod_{j=1}^{n}\left(1-w_{\widetilde{A}_{j}}\right)^{\tau_{j}} \geq \prod_{j=1}^{n}\left(1-\bar{w}_{\widetilde{A}_{j}}\right)^{\tau_{j}}, \\
& \quad \tau_{j} \geq 0, \quad \forall j, \\
& \Rightarrow 1-\prod_{j=1}^{n}\left(1-w_{\widetilde{A}_{j}}\right)^{\tau_{j}} \leq 1-\prod_{j=1}^{n}\left(1-\bar{w}_{\widetilde{A}_{j}}\right)^{\tau_{j}}, \\
& \forall j, \quad \text { and } \\
& u_{\widetilde{A}_{j}} \geq \bar{u}_{\widetilde{A}_{j}} \Rightarrow u_{\widetilde{A}_{j}}^{\tau_{j}} \geq \bar{u}_{\widetilde{A}_{j}}^{\tau_{j}}, \quad \tau_{j} \geq 0, \quad \forall j,
\end{aligned}
$$

$$
\Rightarrow \prod_{j=1}^{n} u_{\widetilde{A}_{j}}^{\tau_{j}} \geq \prod_{j=1}^{n} \bar{u}_{\widetilde{A}_{j}}^{\tau_{j}}, \quad \forall j .
$$

Hence

$$
\begin{aligned}
& {\left[\left(\frac{1}{\sum_{j=1}^{n} \frac{\tau_{j}}{a_{j}}}, \frac{1}{\sum_{j=1}^{n} \frac{\tau_{j}}{b_{j}}}, \frac{1}{\sum_{j=1}^{n} \frac{\tau_{j}}{c_{j}}}, \frac{1}{\sum_{j=1}^{n} \frac{\tau_{j}}{d_{j}}}\right) ;\right.} \\
& \left.1-\prod_{j}^{n}\left(1-w_{\widetilde{A}_{j}}\right)^{\tau_{j}}, \prod_{j}^{n} u_{\widetilde{A}_{j}}^{\tau_{j}}\right] \\
& \leq\left[\left(\frac{1}{\sum_{j=1}^{n} \frac{\tau_{j}}{\bar{a}_{j}}}, \frac{1}{\sum_{j=1}^{n} \frac{\tau_{j}}{\bar{b}_{j}}}, \frac{1}{\sum_{j=1}^{n} \frac{\tau_{j}}{\bar{c}_{j}}}, \frac{1}{\sum_{j=1}^{n} \frac{\tau_{j}}{\bar{d}_{j}}}\right) ;\right. \\
& \left.1-\prod_{j}^{n}\left(1-\bar{w}_{\widetilde{A}_{j}}\right)^{\tau_{j}}, \prod_{j}^{n} \bar{u}_{\widetilde{A}_{j}}^{\tau_{j}}\right] \\
& \Rightarrow \operatorname{Tr} \operatorname{IFWHM}\left(\widetilde{A}_{1}, \widetilde{A}_{2}, \cdots, \widetilde{A}_{n}\right) \\
& \leq \operatorname{Tr} \operatorname{IFWHM}\left(\overline{\widetilde{A}}_{1}, \overline{\widetilde{A}}_{2}, \cdots, \overline{\widetilde{A}}_{n}\right) .
\end{aligned}
$$

(P3) Boundedness: Let $\widetilde{A}_{j}(j=1,2, \cdots, n)$ be a collection of positive TrIFNs and let

$$
\begin{aligned}
\tilde{A}^{-}= & {\left[\left(\min _{j}\left\{a_{j}\right\}, \min _{j}\left\{b_{j}\right\}, \min _{j}\left\{c_{j}\right\}, \min _{j}\left\{d_{j}\right\}\right) ;\right.} \\
& \left.\min _{j}\left\{w_{\widetilde{A}_{j}}\right\}, \max _{j}\left\{u_{\widetilde{A}_{j}}\right\}\right], \\
\widetilde{A}^{+}= & {\left[\left(\max _{j}\left\{a_{j}\right\}, \max _{j}\left\{b_{j}\right\}, \max _{j}\left\{c_{j}\right\}, \max _{j}\left\{d_{j}\right\}\right) ;\right.} \\
& \left.\max _{j}\left\{w_{\widetilde{A}_{j}}\right\}, \min _{j}\left\{u_{\widetilde{A}_{j}}\right\}\right] .
\end{aligned}
$$

Then

$$
\widetilde{A}^{-} \leq \operatorname{Tr} \operatorname{IFWHM}\left(\widetilde{A}_{1}, \widetilde{A}_{2}, \cdots, \widetilde{A}_{n}\right) \leq \widetilde{A}^{+} .
$$

Proof: Boundedness is the consequence of idempotency and monotonicity.

Now, on the basis of Definition 4 and OWH operator [8], we define Trapezoidal Intuitionistic Fuzzy Ordered Weighted Harmonic Mean (TrIFOWHM) operator.

Definition 10. Let $\widetilde{A}_{j}(j=1,2, \cdots, n)$ be a collection of positive TrIFNs. A TrIFOWHM operator is a mapping TrIFOWHM : $\mho^{n} \rightarrow \mho$ which is defined by:

$$
\operatorname{Tr} \operatorname{IFOWHM}\left(\widetilde{A}_{1}, \widetilde{A}_{2}, \cdots, \widetilde{A}_{n}\right)=\frac{1}{\sum_{j=1}^{n} \frac{t_{j}}{A_{\rho(j)}}},
$$


where $t=\left(t_{1}, t_{2}, \cdots, t_{n}\right)^{T}$ is an associated weight vector and $t_{j} \in[0,1], \sum_{j=1}^{n} t_{j}=1 .\left(\rho_{1}, \rho_{2}, \cdots, \rho_{n}\right)$ is any permutation of $(1,2, \cdots, n)$ and $\widetilde{A}_{\rho(j)} \leq \widetilde{A}_{\rho(j-1)}, \forall j=$ $2,3, \cdots, n$.

Theorem 2. Let $\widetilde{A}_{j}(j=1,2, \cdots, n)$ be a collection of positive TrIFNs and $t=\left(t_{1}, t_{2}, \cdots, t_{n}\right)^{T}$ be an associated weight vector where $t_{j} \in[0,1], \sum_{j=1}^{n} t_{j}=$ 1. Then, the aggregated result of $\widetilde{A}_{j}$ by using the TrIFOWHM operator is still a TrIFN, i.e.:

$$
\begin{aligned}
& \operatorname{TrIFOWHM}\left(\widetilde{A}_{1}, \widetilde{A}_{2}, \cdots, \widetilde{A}_{n}\right) \\
&=\left[\left(\frac{1}{\sum_{j=1}^{n} \frac{t_{j}}{a_{\rho(j)}}}, \frac{1}{\sum_{j=1}^{n} \frac{t_{j}}{b_{\rho(j)}}}, \frac{1}{\sum_{j=1}^{n} \frac{t_{j}}{c_{\rho(j)}}}, \frac{1}{\sum_{j=1}^{n} \frac{t_{j}}{d_{\rho(j)}}}\right)\right. \\
&\left.1-\prod_{j}^{n}\left(1-w_{\widetilde{A}_{\rho(j)}}\right)^{t_{j}}, \prod_{j}^{n} u_{\widetilde{A}_{\rho(j)}}^{t_{j}}\right]
\end{aligned}
$$

where $\left(\rho_{1}, \rho_{2}, \cdots, \rho_{n}\right)$ is a permutation of $(1,2, \cdots, n)$ and $\widetilde{A}_{\rho(j)} \leq \widetilde{A}_{\rho(j-1)}, \forall j=2,3, \cdots, n$.

Theorem 3. Let $\widetilde{A}_{j}(j=1,2, \cdots, n)$ be a collection of positive TrIFNs and $t=\left(t_{1}, t_{2}, \cdots, t_{n}\right)^{T}$ be the associated weight vector and $t_{j} \in[0,1], \sum_{j=1}^{n} t_{j}=1$. Then, TrIFOWHM satisfies the following properties:

(P1) Idempotency: If all input arguments $\widetilde{A}_{j}$ are equal and $\widetilde{A}_{j}=A=\left[(a, b, c, d) ; w_{\tilde{A}}, u_{\widetilde{A}}\right], \forall j$. Then:

$$
\begin{aligned}
& \operatorname{Tr} \operatorname{IFOWHM}\left(\widetilde{A}_{1}, \widetilde{A}_{2}, \cdots, \widetilde{A}_{n}\right) \\
& \quad=\operatorname{Tr} \operatorname{IFOWHM}(\widetilde{A}, \widetilde{A}, \cdots, \widetilde{A})=\widetilde{A}
\end{aligned}
$$

(P2) Monotonicity: Let $\widetilde{A}_{j}=\left[\left(a_{j}, b_{j}, c_{j}, d_{j}\right) ; w_{\tilde{A}_{j}}\right.$, $\left.u_{\widetilde{A}_{j}}\right]$ and $\bar{A}_{j}=\left[\left(\bar{a}_{j}, \bar{b}_{j}, \bar{c}_{j}, \bar{d}_{j}\right) ; \bar{w}_{\widetilde{A}_{j}}, \bar{u}_{\widetilde{A}_{j}}\right](j=1,2, \cdots$, $n)$ be two collections of positive TrIFNs. If $a_{j} \leq \bar{a}_{j}$, $b_{j} \leq \bar{b}_{j}, c_{j} \leq \bar{c}_{j}, d_{j} \leq \bar{d}_{j}, w_{\widetilde{A}_{j}} \leq \bar{w}_{\widetilde{A}_{j}}$ and $u_{\widetilde{A}_{j}} \geq \bar{u}_{\widetilde{A}_{j}}$, $\forall j$, then

$$
\begin{aligned}
& \operatorname{Tr} \operatorname{IFOWHM}\left(\widetilde{A}_{1}, \widetilde{A}_{2}, \cdots, \widetilde{A}_{n}\right) \\
& \leq \operatorname{Tr} \operatorname{IFOWHM}\left(\overline{\widetilde{A}}_{1}, \overline{\widetilde{A}}_{2}, \cdots, \overline{\widetilde{A}}_{n}\right) .
\end{aligned}
$$

(P3) Boundedness: Let $\widetilde{A}_{j}(j=1,2, \cdots, n)$ be any collection of positive TrIFNs and then

$$
\widetilde{A}^{-} \leq \operatorname{Tr} \operatorname{IFOWHM}\left(\widetilde{A}_{1}, \widetilde{A}_{2}, \cdots, \widetilde{A}_{n}\right) \leq \widetilde{A}^{+} .
$$

where

$$
\begin{aligned}
\widetilde{A}^{-}= & {\left[\left(\min _{j}\left\{a_{j}\right\}, \min _{j}\left\{b_{j}\right\}, \min _{j}\left\{c_{j}\right\}, \min _{j}\left\{d_{j}\right\}\right) ;\right.} \\
& \left.\min _{j}\left\{w_{\widetilde{A}_{j}}\right\}, \max _{j}\left\{u_{\widetilde{A}_{j}}\right\}\right], \\
\widetilde{A}^{+}= & {\left[\max _{j}\left\{a_{j}\right\}, \max _{j}\left\{b_{j}\right\}, \max _{j}\left\{c_{j}\right\}, \max _{j}\left\{d_{j}\right\}\right) ; } \\
& \left.\max _{j}\left\{w_{\widetilde{A}_{j}}\right\}, \min _{j}\left\{u_{\widetilde{A}_{j}}\right\}\right] .
\end{aligned}
$$

(P4) Commutativity: If $\left\{\widetilde{B}_{1}, \widetilde{B}_{2}, \cdots, \widetilde{B}_{n}\right\}$ is any permutation of $\left\{\widetilde{A}_{1}, \widetilde{A}_{2}, \cdots, \widetilde{A}_{n}\right\}$, then

$$
\begin{aligned}
& \operatorname{TrIFOWHM}\left\{\widetilde{A}_{1}, \widetilde{A}_{2}, \cdots, \widetilde{A}_{n}\right\} \\
& =\operatorname{TrIFOWHM}\left\{\widetilde{B}_{1}, \widetilde{B}_{2}, \cdots, \widetilde{B}_{n}\right\} .
\end{aligned}
$$

Remark 1. It is to be noted that TrIFWHM operator (Eq. (7)) does not have commutative property, but operator TrIFOWHM (Eq. (11)) has commutative property. Moreover, depending on the associate weight vector, $t$, few special cases of the TrIFOWHM operator are analyzed below:

- If $t=(1,0, \cdots, 0)^{T}$, then

$$
\operatorname{TrIFOWHM}\left\{\widetilde{A}_{1}, \widetilde{A}_{2}, \cdots, \widetilde{A}_{n}\right\}=\max _{j}\left\{\widetilde{A}_{j}\right\} .
$$

- If $t=(0,0, \cdots, 1)^{T}$, then

$$
\operatorname{TrIFOWHM}\left\{\widetilde{A}_{1}, \widetilde{A}_{2}, \cdots, \widetilde{A}_{n}\right\}=\min _{j}\left\{\widetilde{A}_{j}\right\} .
$$

- If $t_{j}=1$ and $t_{i}=0, i \neq j$, then

$$
\operatorname{TrIFOWHM}\left\{\widetilde{A}_{1}, \widetilde{A}_{2}, \cdots, \widetilde{A}_{n}\right\}=\widetilde{A}_{\rho(j)},
$$

where $\widetilde{A}_{\rho(j)}$ is the $j$ th largest $\operatorname{TrIFN} \widetilde{A}_{j}(j=$ $1,2, \cdots, n)$.

- If $t=\left(\frac{1}{n}, \frac{1}{n}, \cdots, \frac{1}{n}\right)^{T}$, then:

$$
\begin{aligned}
& \operatorname{TrIFOWHM}\left\{\widetilde{A}_{1}, \widetilde{A}_{2}, \cdots, \widetilde{A}_{n}\right\} \\
& =\operatorname{Tr} \operatorname{IFHM}\left\{\widetilde{A}_{1}, \widetilde{A}_{2}, \cdots, \widetilde{A}_{n}\right\} .
\end{aligned}
$$

In the following, Trapezoidal Intuitionistic Fuzzy Induced OWH Mean (TrIFIOWHM) operator is presented.

Definition 11. Let $\widetilde{A}_{j}(j=1,2, \cdots, n)$ be a collection of positive TrIFNs and $t=\left(t_{1}, t_{2}, \cdots, t_{n}\right)^{T}$ be an associated weight vector and $t_{j} \in[0,1], \sum_{j=1}^{n} t_{j}=1$. A TrIFIOWHM operator is defined by: 


$$
\begin{aligned}
& \operatorname{TrIFIOWHM}\left[\left(U_{1}, \widetilde{A}_{1}\right),\left(U_{2}, \widetilde{A}_{2}\right), \cdots,\left(U_{n}, \widetilde{A}_{n}\right)\right] \\
& \quad=\frac{1}{\sum_{j=1}^{n} \frac{t_{j}}{\widetilde{B}_{j}}}
\end{aligned}
$$

where $\widetilde{B}_{j}=\left[\left(e_{j}, f_{j}, g_{j}, h_{j}\right) ; w_{\widetilde{B}_{j}}^{\prime}, u_{\widetilde{B}_{j}}^{\prime}\right]$ is $\widetilde{A}_{i}$ value of the TrIFIOWHM pair $\left(U_{i}, \widetilde{A}_{i}\right)$ having the $j$ th largest $U_{i}$. In $\left(U_{i}, \widetilde{A}_{i}\right), U_{i}$ is known as the order-inducing variable, and $\widetilde{A}_{i}$ is known as the TrIFNs argument variable.

Theorem 4. Let $\widetilde{A}_{j}(j=1,2, \cdots, n)$ be a collection of positive TrIFNs and then their aggregation result by using the TrIFIOWHM operator be also a $\operatorname{TrIFN}$, i.e.:

$$
\begin{aligned}
& \operatorname{Tr} I F I O W H M\left(\widetilde{A}_{1}, \widetilde{A}_{2}, \cdots, \widetilde{A}_{n}\right) \\
&=\left[\left(\frac{1}{\sum_{j=1}^{n} \frac{t_{j}}{e_{j}}}, \frac{1}{\sum_{j=1}^{n} \frac{t_{j}}{f_{j}}}, \frac{1}{\sum_{j=1}^{n} \frac{t_{j}}{g_{j}}}, \frac{1}{\sum_{j=1}^{n} \frac{t_{j}}{h_{j}}}\right) ;\right. \\
&\left.1-\prod_{j}^{n}\left(1-w_{\widetilde{B}_{j}}^{\prime}\right)^{t_{j}}, \prod_{j}^{n} u_{\widetilde{B}_{j}}{ }^{t_{j}}\right]
\end{aligned}
$$

where $t=\left(t_{1}, t_{2}, \ldots, t_{n}\right)^{T}$ is an associated weight vector and $t_{j} \in[0,1], \sum_{j=1}^{n} t_{j}=1$.

Theorem 5. Let $\widetilde{A}_{j}(j=1,2, \cdots, n)$ be a collection of positive $\operatorname{TrIFNs}$ and $t=\left(t_{1}, t_{2}, \cdots, t_{n}\right)^{T}$ be an associated weight vector and $t_{j} \in[0,1], \sum_{j=1}^{n} t_{j}=1$. Then, the operator TrIFIOWHM satisfies idempotency, monotonicity, and commutativity properties.

It is known that the TrIFWHM operator reflects the importance of individual input and the TrIFOWHM gives the importance to their ordered positions. Now, we introduce a new operator, Trapezoidal Intuitionistic Fuzzy hybrid Harmonic Mean (TrIFhHM) operator, which lends the importance to both of the given inputs as well as their ordered positions.

Definition 12. Let $\widetilde{A}_{j}(j=1,2, \cdots, n)$ be a collection of positive TrIFNs and $t=\left(t_{1}, t_{2}, \cdots, t_{n}\right)^{T}$ be associated weight vector where $t_{j} \in[0,1], \sum_{j=1}^{n} t_{j}=1$. A TrIFhHM operator of $n$ dimension is a mapping TrIFhHM : $\mho^{n} \rightarrow \mho$ which is defined by:

$$
\operatorname{Tr} \operatorname{IFhHM}\left(\widetilde{A}_{1}, \widetilde{A}_{2}, \cdots, \widetilde{A}_{n}\right)=\frac{1}{\sum_{j=1}^{n} \frac{t_{j}}{\widetilde{A}_{\rho(j)}^{\prime}}}
$$

where $A_{\rho(j)}^{\prime}$ is the $j$ th largest of weighted TrIFNs $\widetilde{A}_{j}^{\prime}\left(\widetilde{A}_{j}^{\prime}=n \tau_{j} \widetilde{A}_{j}, j=1,2, \cdots, n\right)$. Here, $n$ is called balancing coefficient, and $\tau=\left(\tau_{1}, \tau_{2}, \cdots, \tau_{n}\right)^{T}$ is the weight vector of $\widetilde{A}_{j}(j=1,2, \cdots, n)$ and $\tau_{j} \in[0,1]$, $\sum_{j=1}^{n} \tau_{j}=1$ and $\left(\rho_{1}, \rho_{2}, \cdots, \rho_{n}\right)$ is a permutation of $(1,2, \cdots, n), \forall j=1,2, \cdots, n$.

Theorem 6. Let $\widetilde{A}_{j}(j=1,2, \cdots, n)$ be a collection of positive TrIFNs and $t=\left(t_{1}, t_{2}, \cdots, t_{n}\right)^{T}$ be an associated weight vector where $t_{j} \in[0,1], \sum_{j=1}^{n} t_{j}=1$. Then, their aggregation result by using the TrIFhHM operator is also a $\operatorname{TrIFN}$, i.e.:

$$
\begin{aligned}
& \operatorname{Tr} I F h H M\left(\widetilde{A}_{1}, \widetilde{A}_{2}, \cdots, \widetilde{A}_{n}\right) \\
&=\left[\left(\frac{1}{\sum_{j=1}^{n} \frac{t_{j}}{a_{\rho(j)}^{\prime}}}, \frac{1}{\sum_{j=1}^{n} \frac{t_{j}}{b_{\rho(j)}^{\prime}}}, \frac{1}{\sum_{j=1}^{n} \frac{t_{j}}{c_{\rho(j)}^{\prime}}}, \frac{1}{\sum_{j=1}^{n} \frac{t_{j}}{d_{\rho(j)}^{\prime}}}\right)\right. \\
&\left.1-\prod_{j}^{n}\left(1-w_{\widetilde{A}_{\rho(j)}^{\prime}}^{\prime}\right)^{t_{j}}, \prod_{j}^{n} u_{\widetilde{A}_{\rho(j)}^{\prime}}^{t_{j}}\right]
\end{aligned}
$$

Remark 2. In the following, depending on the weight vectors, few special cases of the TrIFhHM operator are analyzed:

- If $\tau=\left(\tau_{1}, \tau_{2}, \cdots, \tau_{n}\right)^{T}$ approaches $\tau=\left(\frac{1}{n}, \frac{1}{n}, \ldots\right.$, $\left.\frac{1}{n}\right)^{T}$, then vector $\left(\widetilde{A}_{\rho(1)}^{\prime}, \widetilde{A}_{\rho(2)}^{\prime}, \cdots, \widetilde{A}_{\rho(n)}^{\prime}\right)$ approaches $\left(\widetilde{A}_{\rho(1)}, \widetilde{A}_{\rho(2)}, \cdots, \widetilde{A}_{\rho(n)}\right)$ and the operator TrIFhHM reduces to TrIFOWHM operator (Eq. (11));

- If $t=\left(t_{1}, t_{2}, \cdots, t_{n}\right)^{T}$ approaches $t=\left(\frac{1}{n}, \frac{1}{n}, \ldots\right.$, $\left.\frac{1}{n}\right)^{T}$, then $\left(\widetilde{A}_{\rho(1)}^{\prime}, \widetilde{A}_{\rho(2)}^{\prime}, \cdots, \widetilde{A}_{\rho(n)}^{\prime}\right)$ approaches $\left(\widetilde{A}_{1}\right.$, $\left.\widetilde{A}_{2}, \cdots, \widetilde{A}_{n}\right)$ and the operator TrIFhHM reduces to TrIFWHM operator (Eq. (7)).

\section{An application to group decision making problem with TrIFNs information}

With the increasing complexity in real-life decision making scenario, it is usually a hard task for a single expert to evaluate all the relevant issues correctly. Thus, in order to improve the rationality and reliability of the decisive result, many real-world processes take place by a group of experts. With this view point, a group decision making problem is presented where the information is quantified by TrIFNs. Let the MAGDM problem be designed by $m$ alternatives $\mathbb{A}=\left\{\mathbb{A}_{1}, \mathbb{A}_{2}, \cdots, \mathbb{A}_{m}\right\}$. The expert unit assesses these $m$ alternatives with respect to $n$ attributes $\mathcal{C}=$ $\left\{\mathcal{C}_{1}, \mathcal{C}_{2}, \cdots, \mathcal{C}_{n}\right\}$ with their corresponding weights $\tau=$ $\left(\tau_{1}, \tau_{2}, \cdots, \tau_{n}\right)^{T}$, where $\tau_{j} \in[0,1], j=1,2, \cdots, n$ and 
$\sum_{j}^{n} \tau_{j}=1$. The set of attributes $\mathcal{C}$ can be classified into two subsets: the subset of benefit attributes $P$ and the subset of cost attributes $Q$ with $P \cup Q=\mathcal{C}$, $P \cap Q=\phi$. The characteristic of benefit attribute is the larger, the better and that of cost attribute is the smaller, the better. Let $E=\left\{E_{1}, E_{2}, \cdots, E_{k}\right\}$ be the set of $k$ experts and $\eta=\left(\eta_{1}, \eta_{2}, \cdots, \eta_{k}\right)^{T}$ be the weight vector of these $k$ experts, where:

$$
\eta_{l} \in[0,1], l=1,2, \cdots, k \text { and } \sum_{l=1}^{k} \eta_{l}=1 .
$$

The TrIFWHM operator and TrIFhHM-based approach to solve MAGDM problem can be described in the following steps:

- Step 1: Construction of decision matrix. The MAGDM problem with TrIFNs can be given in the form of decision matrix $D_{t}=\left(\widetilde{A}_{i j}^{l}\right)_{m \times n}$, provided by decision maker $E_{l}$ as follows:

$$
D_{l}=\left(\widetilde{A}_{i j}^{l}\right)_{m \times n}=\mathbb{A}_{1}\left(\begin{array}{cccc}
\mathbb{C}_{1} & \mathcal{C}_{2} & \cdots & \mathcal{C}_{n} \\
\widetilde{A}_{11}^{l} & \widetilde{A}_{12}^{l} & \cdots & \widetilde{A}_{1 n}^{l} \\
\widetilde{A}_{21}^{l} & \widetilde{A}_{22}^{l} & \cdots & \widetilde{A}_{2 n}^{l} \\
\vdots & \vdots & \cdots & \vdots \\
\widetilde{A}_{m 1}^{l} & \widetilde{A}_{m 2}^{l} & \cdots & \widetilde{A}_{m n}^{l}
\end{array}\right) .
$$

where:

$$
\begin{aligned}
& \widetilde{A}_{i j}^{l}=\left[\left(a_{i j}^{l}, b_{i j}^{l}, c_{i j}^{l}, d_{i j}^{l}\right) ; w_{\widetilde{A}_{i j}}^{l}, u_{\widetilde{A}_{i j}}^{l}\right] \\
& (l=1,2, \cdots, k) .
\end{aligned}
$$

- Step 2: Normalization of the decision matrix. To avoid the effect of different attributes' scale on decisive results, the primary task is to transform the decision matrix into the normalized decision matrix:

$$
N_{l}=\left(\widetilde{r}_{i j}^{l}\right)_{m \times n}=\mathbb{A}_{\mathbb{A}_{1}}\left(\begin{array}{cccc}
C_{1} & \mathcal{C}_{2} & \cdots & \mathcal{C}_{n} \\
\widetilde{r}_{11}^{l} & \widetilde{r}_{12}^{l} & \cdots & \widetilde{r}_{1 n}^{l} \\
\widetilde{r}_{21}^{l} & \widetilde{r}_{22}^{l} & \cdots & \widetilde{r}_{2 n}^{l} \\
\vdots & \vdots & \cdots & \vdots \\
\widetilde{r}_{m 1}^{l} & \widetilde{r}_{m 2}^{l} & \cdots & \widetilde{r}_{m n}^{l}
\end{array}\right),
$$

where each $\widetilde{r}_{i j}^{i}$ can be computed by the following formulae:

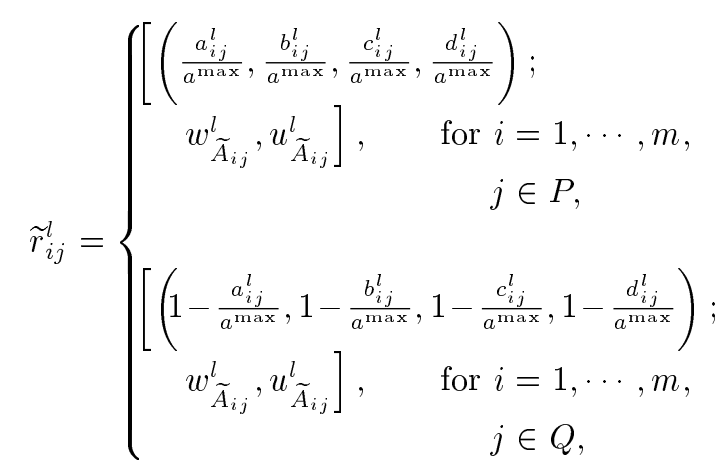

where:

$$
\begin{aligned}
a^{\max } & =\max _{i, j}\left\{a_{i j}^{l}, b_{i j}^{l}, c_{i j}^{l}, d_{i j}^{l}\right\}, \\
i & =1,2, \cdots, m, \quad j=1,2, \cdots, n .
\end{aligned}
$$

For the sake of simplicity, it can be written as:

$$
\widetilde{r}_{i j}^{l}=\left[\left(r_{i j}^{1 l}, r_{i j}^{2 l}, r_{i l}^{3 t}, r_{i j}^{4 l}\right) ; w_{\widetilde{A}_{i j}}^{l}, u_{\widetilde{A}_{i j}}^{l}\right] .
$$

- Step 3: Computation of the individual overall ratings of all the alternatives. To derive the individual overall ratings of all the alternatives, the TrIFWHM operator is applied and:

$$
\begin{aligned}
\widetilde{R}_{i}^{l} & =\operatorname{TrIFWHM}\left(\widetilde{r}_{i 1}^{l}, \widetilde{r}_{i 2}^{l}, \cdots, \widetilde{r}_{i n}^{l}\right), \\
i & =1,2, \cdots, m ; \quad l=1,2, \cdots, k .
\end{aligned}
$$

- Step 4: Computation of the collective overall ratings of all the alternatives. The collective overall ratings of all the alternatives are derived by utilizing TrIFhHM operator:

$$
\begin{aligned}
\widetilde{R}_{i} & =\operatorname{TrIFhHM}\left(\widetilde{R}_{i}^{1}, \widetilde{R}_{i}^{2}, \cdots, \widetilde{R}_{i}^{k}\right) \\
& =\left[\left(a_{i}, b_{i}, c_{i}, d_{i}\right) ; w_{\widetilde{R}_{i}}, u_{\widetilde{R}_{i}}\right] .
\end{aligned}
$$

- Step 5: Ranking of the alternatives. Ranking is done according to the ranking process defined in Section 2.2.

- Step 6: End.

\section{Enterprise selection example}

In this section, to illustrate the application of the proposed operators, we consider a MAGDM problem adopted from [54].

A company wants to form a cooperative alliance with some potential enterprises to fulfill the market demand. After pre-evaluation, three enterprises, $\mathbb{A}_{i}(i=1,2,3)$, are selected for further evaluation. The expert unit selects the best enterprises on the basis of the following four attributes: producing ability $\mathcal{C}_{1}$, the technology capability $\mathcal{C}_{2}$, capital currency $\mathcal{C}_{3}$, and 
research ability $\mathcal{C}_{4}$. Let $\tau=(0.15,0.35,0.3,0.2)^{T}$ be the weight vector of these four attributes. In the expert committee, there are three experts $E_{1}, E_{2}$, and $E_{3}$, whose corresponding weight vector is $\eta=$ $(0.35,0.35,0.30)^{T}$.

Now, we shall utilize the TrIFWHM and the TrIFhHm operators to propose an approach to solve above enterprise selection problem with intuitionistic fuzzy information as follows:
- Step 1: The evaluation of alternatives (enterprises) against all the attributes is given in Tables 1-3;

- Step 2: The above criteria are benefit criteria. So, the normalized decision matrices, computed by utilizing Eq. (17), are provided in Tables 4 to 6;

- Step 3: The individual overall ratings (TrIFNs) of all alternatives, $\mathbb{A}_{i}$, by using TrIFWHM operator with $\tau=(0.15,0.35,0.3,0.2)^{T}$ are computed and

Table 1. Decision matrix provided by expert $E_{1}$.

\begin{tabular}{ccccc}
\hline Alter. $^{*}$ & $\mathcal{C}_{\mathbf{1}}$ & $\mathcal{C}_{\mathbf{2}}$ & $\mathcal{C}_{\boldsymbol{3}}$ & $\mathcal{C}_{\mathbf{4}}$ \\
\hline $\mathbb{A}_{\mathbf{1}}$ & {$[(2,4,6,8) ; 0.5,0.4]$} & {$[(2,4,6,7) ; 0.7,0.2]$} & {$[(17,18,19,20) ; 0.6,0.3]$} & {$[(3,4,6,7) ; 0.7,0.1]$} \\
$\mathbb{A}_{\mathbf{2}}$ & {$[(3,5,6,7) ; 0.6,0.3]$} & {$[(15,17,19,20) ; 0.7,0.2]$} & {$[(3,4,5,6) ; 0.7,0.2]$} & {$[(4,5,6,7) ; 0.6,0.4]$} \\
$\mathbb{A}_{\mathbf{3}}$ & {$[(1,2,3,4) ; 0.7,0.2]$} & {$[(2,3,4,5) ; 0.5,0.4]$} & {$[(2,4,5,6) ; 0.6,0.4]$} & {$[(15,16,18,20) ; 0.8,0.1]$} \\
\hline
\end{tabular}

*Alter.: Alternative

Table 2. Decision matrix provided by expert $E_{2}$.

\begin{tabular}{ccccc}
\hline Alter. ${ }^{*}$ & $\mathcal{C}_{\boldsymbol{1}}$ & $\mathcal{C}_{\mathbf{2}}$ & $\mathcal{C}_{\mathbf{3}}$ & $\mathcal{C}_{4}$ \\
\hline $\mathbb{A}_{\mathbf{1}}$ & {$[(15,16,17,20) ; 0.9,0.1]$} & {$[(2,4,5,7) ; 0.5,0.3]$} & {$[(2,5,6,8) ; 0.7,0.2]$} & {$[(3,5,6,7) ; 0.8,0.1]$} \\
$\mathbb{A}_{\mathbf{2}}$ & {$[(4,5,6,7) ; 0.6,0.3]$} & {$[(16,17,19,20) ; 0.8,0.2]$} & {$[(3,4,5,6) ; 0.7,0.2]$} & {$[(4,5,6,7) ; 0.6,0.3]$} \\
$\mathbb{A}_{\mathbf{3}}$ & {$[(1,3,5,6) ; 0.6,0.4]$} & {$[(2,3,4,6) ; 0.6,0.3]$} & {$[(2,3,4,5) ; 0.6,0.4]$} & {$[(17,18,19,20) ; 0.6,0.3]$} \\
\hline
\end{tabular}

${ }^{*}$ Alter.: Alternative

Table 3. Decision matrix provided by expert $E_{3}$.

\begin{tabular}{ccccc}
\hline Alter. $^{*}$ & $\mathcal{C}_{\mathbf{1}}$ & $\mathcal{C}_{\mathbf{2}}$ & $\mathcal{C}_{\mathbf{3}}$ & $\mathcal{C}_{4}$ \\
\hline $\mathbb{A}_{\mathbf{1}}$ & {$[(4,5,6,8) ; 0.5,0.4]$} & {$[(1,2,3,4) ; 0.7,0.2]$} & {$[(17,18,19,20) ; 0.6,0.25]$} & {$[(3,4,5,6) ; 0.7,0.1]$} \\
$\mathbb{A}_{\mathbf{2}}$ & {$[(3,5,6,7) ; 0.6,0.2]$} & {$[(2,3,4,6) ; 0.6,0.3]$} & {$[(3,4,5,6) ; 0.7,0.2]$} & {$[(16,17,19,20) ; 0.8,0.2]$} \\
$\mathbb{A}_{\mathbf{3}}$ & {$[(16,17,18,20) ; 0.8,0.1]$} & {$[(4,5,6,7) ; 0.5,0.4]$} & {$[(2,4,5,6) ; 0.6,0.4]$} & {$[(3,4,6,7) ; 0.7,0.2]$} \\
\hline
\end{tabular}

*Alter.: Alternative

Table 4. Normalized decision matrix provided by expert $E_{1}$.

\begin{tabular}{ccccc}
\hline Alter. $^{*}$ & $\mathcal{C}_{\mathbf{1}}$ & $\mathcal{C}_{\mathbf{2}}$ & $\mathcal{C}_{3}$ & $\mathcal{C}_{4}$ \\
\hline $\mathbb{A}_{\mathbf{1}}$ & {$[(0.1,0.2,0.3,0.4) ; 0.5,0.4]$} & {$[(0.1,0.2,0.3,0.35) ; 0.7,0.2]$} & {$[(0.85,0.9,0.95,1) ; 0.6,0.3]$} & {$[(0.15,0.2,0.3,0.35) ; 0.7,0.1]$} \\
$\mathbb{A}_{\mathbf{2}}$ & {$[(0.15,0.25,0.3,0.35) ; 0.6,0.3]$} & {$[(0.75,0.85,0.95,1) ; 0.7,0.2]$} & {$[(0.15,0.2,0.25,0.3) ; 0.7,0.2][(0.2,0.25,0.3,0.35) ; 0.6,0.4]$} \\
$\mathbb{A}_{\mathbf{3}}$ & {$[(0.05,0.1,0.15,0.2) ; 0.7,0.2]$} & {$[(0.1,0.15,0.2,0.25) ; 0.5,0.4]$} & {$[(0.1,0.2,0.25,0.3) ; 0.6,0.4]$} & {$[(0.75,0.8,0.9,1) ; 0.8,0.1]$} \\
\hline
\end{tabular}

*Alter.: Alternative

Table 5. Normalized decision matrix provided by expert $E_{2}$.

\begin{tabular}{cccc}
\hline Alter. $^{*}$ & $\mathcal{C}_{1}$ & $\mathcal{C}_{\mathbf{2}}$ & $\mathcal{C}_{\mathbf{3}}$ \\
\hline $\mathbb{A}_{\mathbf{1}}$ & {$[(0.75,0.8,0.85,1.0) ; 0.9,0.1][(0.1,0.2,0.25,0.35) ; 0.5,0.3][(0.1,0.25,0.3,0.4) ; 0.7,0.2][(0.15,0.25,0.3,0.35) ; 0.8,0.1]$} \\
$\mathbb{A}_{\mathbf{2}}$ & {$[(0.2,0.25,0.3,0.35) ; 0.6,0.3][(0.8,0.85,0.95,1) ; 0.8,0.2][(0.15,0.2,0.25,0.3) ; 0.7,0.2][(0.2,0.25,0.3,0.35) ; 0.6,0.3]$} \\
$\mathbb{A}_{\mathbf{3}}$ & {$[(0.05,0.15,0.25,0.3) ; 0.6,0.4][(0.1,0.15,0.2,0.3) ; 0.6,0.3][(0.1,0.15,0.2,0.25) ; 0.6,0.4][(0.85,0.90,0.95,1) ; 0.6,0.3]$} \\
\hline
\end{tabular}

*Alter.: Alternative

Table 6. Normalized decision matrix provided by expert $E_{3}$.

\begin{tabular}{cccc}
\hline Alter. $^{*}$ & $\mathcal{C}_{\mathbf{1}}$ & $\mathcal{C}_{\mathbf{2}}$ & $\mathcal{C}_{\mathbf{3}}$ \\
\hline $\mathbb{A}_{\mathbf{1}}$ & {$[(0.2,0.25,0.3,0.4) ; 0.5,0.4]$} & {$[(0.05,0.1,0.15,0.2) ; 0.7,0.2][(0.85,0.9,0.95,1) ; 0.6,0.25][(0.15,0.2,0.25,0.3) ; 0.7,0.1]$} \\
$\mathbb{A}_{\mathbf{2}}$ & {$[(0.15,0.25,0.3,0.35) ; 0.6,0.2][(0.1,0.15,0.2,0.3) ; 0.6,0.2][(0.15,0.2,0.25,0.3) ; 0.7,0.2][(0.8,0.85,0.95,1.0) ; 0.8,0.2]$} \\
$\mathbb{A}_{\mathbf{3}}$ & {$[(0.8,0.85,0.9,1.0) ; 0.9,0.1]$} & {$[(0.2,0.25,0.3,0.35) ; 0.5,0.4][(0.1,0.2,0.25,0.3) ; 0.6,0.4][(0.15,0.2,0.3,0.35) ; 0.8,0.1]$} \\
\hline
\end{tabular}

*Alter.: Alternative 
shown below. Here, TrIFWHM operator is used as all the decision matrices consist of extreme outliers. It is worth noticing that few outliers (too large data relative to others) exist in Tables 4 to 6 . For this reason, TrIFWHM operator is utilized as a fusion technique to relieve the influence of extreme outliers on the final results:

$$
\begin{aligned}
& \widetilde{R}_{1}^{1}=[(0.1496,0.2609,0.3775,0.4452) ; 0.6469,0.2182], \\
& \widetilde{R}_{2}^{1}=[(0.2239,0.3020,0.3656,0.4255) ; 0.6682,0.2441], \\
& \widetilde{R}_{3}^{1}=[(0.1024,0.1791,0.2397,0.2985) ; 0.6394,0.2732], \\
& \widetilde{R}_{1}^{2}=[(0.1245,0.2540,0.3083,0.4046) ; 0.7195,0.1808], \\
& \widetilde{R}_{2}^{2}=[(0.2388,0.3020,0.3656,0.4255) ; 0.7121,0.2305], \\
& \widetilde{R}_{3}^{2}=[(0.1027,0.1800,0.2463,0.3261) ; 0.6000,0.3415], \\
& \widetilde{R}_{1}^{3}=[(0.1060,0.1840,0.2532,0.3235) ; 0.6469,0.2066], \\
& \widetilde{R}_{2}^{3}=[(0.1481,0.2142,0.2732,0.3578) ; 0.6806,0.2000], \\
& \widetilde{R}_{3}^{3}=[(0.1595,0.2453,0.3125,0.3675) ; 0.6942,0.2462]
\end{aligned}
$$

- Step 4: The collective overall ratings (TrIFNs) of all alternatives, $\mathbb{A}_{i}$, by using TrIFhHM with $\eta=(0.35,0.35,0.30)^{T}$ (experts' weight vector) and $t=(0.25 .0 .4 .0 .3)^{T}$ (associated weight vector) are computed and shown in Table 7;

- Step 5: Finally, the decision results are obtained by the centroid-based ranking method discussed in Section 2.2 and provided in Table 7 .

It is observed from Table 7 that the ranking order of alternatives is $\mathbb{A}_{2}>\mathbb{A}_{1}>\mathbb{A}_{3}$. Hence, the best enterprise is $\mathbb{A}_{2}$.
Now, the obtained result is compared with the existing approaches through the aforementioned enterprise selection problem. Together, one more comparison analysis is drawn where the same problem is solved with IFSs data instead of TrIFNs.

\subsection{Comparison analysis}

\subsubsection{Comparison of performances with the existing} aggregation operators

In this subsection, the above-mentioned enterprise selection problem (described in Section 5) is solved by using different operators, such as weighted power-average operator [54] of TrIFNs, weighted power geometric operator [55] of TrIFNs, and generalized weighted averaging operator [57] for interval-valued trapezoidal intuitionistic fuzzy number to illustrate the applicability of the proposed operators. The individual overall ratings of alternatives by using these aggregation operators and experts' overall ratings of alternatives by using the corresponding hybrid aggregation operators are computed to compare the performance of the proposed harmonic mean operator with the aforementioned aggregation operators. By using the alternatives' final performances, ranking order of the alternatives in each of the cases is calculated and presented in Table 8 .

It is to be observed from Table 8 that the ranking results by utilizing the proposed harmonic operator for TrIFNs, weighted power-average operator [54], and weighted power geometric operator [55] of TrIFNs are the same. IVTrIFGWA [57] operator selects $A_{2}$ as the best alternative; however, ordering of $A_{1}$ and $A_{3}$ is reverse over the other methods. Both weighted power average operator [54] and weighted power geometric operator [55] of TrIFNs focus on assigning low weight to the unfair data (i.e., extreme outliers) by considering relationship among the input TrIFN arguments. They directly do not address outlier information among the TrIFN data sets. In this respect, our proposed TrIFWHM operator focuses on directly the outlier data

Table 7. Final aggregated values and ranking results.

\begin{tabular}{cccc}
\hline Alter.* $^{*}$ & Final aggregation & Centroid point & Ranking order \\
\hline $\mathbb{A}_{\mathbf{1}}$ & $\widetilde{R}_{1}=[(0.1202,0.2209,0.2939,0.3733) ; 0.6786,0.1989]$ & $X_{\widetilde{R}_{1}}=0.2459, Y_{\widetilde{R}_{1}}=0.3511$ & \\
$\mathbb{A}_{\mathbf{2}}$ & $\widetilde{R}_{2}=[(0.1892,0.2587,0.3208,0.3934) ; 0.6903,0.2246]$ & $X_{\widetilde{R}_{2}}=0.2914, Y_{\widetilde{R}_{2}}=0.3551$ & $\mathbb{A}_{2}>\mathbb{A}_{1}>\mathbb{A}_{3}$ \\
$\mathbb{A}_{\mathbf{3}}$ & $\widetilde{R}_{3}=[(0.1180,0.1988,0.2642,0.3307) ; 0.6415,0.2913]$ & $X_{\widetilde{R}_{3}}=0.2236, Y_{\widetilde{R}_{3}}=0.3505$ & \\
\hline
\end{tabular}

*Alter.: Alternative

Table 8. The ranking order of alternatives for different approaches.

\begin{tabular}{llc}
\hline \multicolumn{1}{c}{ Approach } & \multicolumn{1}{c}{ Operator } & Ranking results \\
\hline Wan [54] & Weighted power average & $\mathbb{A}_{2}>\mathbb{A}_{1}>\mathbb{A}_{3}$ \\
Wan and Dong [55] & Weighted power geometric & $\mathbb{A}_{2}>\mathbb{A}_{1}>\mathbb{A}_{3}$ \\
Dong and Wan [57] & IVTrIFGWA & $\mathbb{A}_{2}>\mathbb{A}_{3}>\mathbb{A}_{1}$ \\
Proposed & TrIFWHM & $\mathbb{A}_{2}>\mathbb{A}_{1}>\mathbb{A}_{3}$ \\
\hline
\end{tabular}


Table 9. Final aggregated values and ranking results of IFS model.

\begin{tabular}{cccc}
\hline Alter. $^{*}$ & Final aggregation & Score value & Ranking order \\
\hline $\mathbb{A}_{\mathbf{1}}$ & $R_{1}=(0.6781,0.1989)$ & $\operatorname{Score}\left(R_{1}\right)=0.4792$ & \\
$\mathbb{A}_{\mathbf{2}}$ & $R_{2}=(0.6903,0.2246)$ & $\operatorname{Score}\left(R_{1}\right)=0.4657$ & $\mathbb{A}_{1}>\mathbb{A}_{2}>\mathbb{A}_{3}$ \\
$\mathbb{A}_{\mathbf{3}}$ & $R_{3}=(0.6415,0.2913)$ & $\operatorname{Score}\left(R_{1}\right)=0.3505$ & \\
\hline${ }^{*}$ Alter.: & Alternative & &
\end{tabular}

to relieve the influence of it to the ultimate aggregation results.

In what follows, we compare the proposed model with the intuitionistic fuzzy value model.

\subsubsection{Comparison analysis with IFS-based MAGDM model}

It is owing to fact that IFNs, generalization of IFSs, are defined on the continuous universe of discourse. Thus, it is more convenient to use IFNs rather than IFSs. However, to make a comparative analysis, we use IFSs to express expert's opinion by considering expert's satisfaction and dissatisfaction degrees (i.e., $\left(w_{i j}, u_{i j}\right)$ ), actually modeled by TrIFNs $\mathbb{A}_{i}(i=1,2,3)$. Then, the weighted intuitionistic fuzzy arithmetic mean operator [29] is used to compute the alternatives' overall ratings. The final performance values of alternatives $\mathbb{A}_{i}(i=1,2,3)$ are computed and given in Table 9 .

Finally, the decision results are obtained by using score function [62]. From Table 9, it is to be noted that enterprise $\mathbb{A}_{1}$ is the best choice.

It is worth noticing that the resultant ranking result is different from the ranking order found by the proposed method (TrIFNs model). This change is due to the transformation of original information (TrIFNs) into intuitionistic fuzzy values by discarding trapezoidal fuzzy numbers from the corresponding TrIFNs. As a result, TrIFN loses its inherent structure. Such a transformation distorts experts' original opinions as well as weakens the ability of information representation of TrIFNs. it is due to the fact that TrIFNs provide a suitable framework to maintain the integrity in information processing in a decision-making problem, compared to IFSs. Hence, MAGDM problems with imprecise information, represented by TrIFNs, provide more accurate results.

\section{Conclusion}

In this study, we have developed a series of aggregation operators (TrIFWHM, TrIFOWHM, TrIFIOWHM, and TrIFhHM) on the basis of HM operator to aggregate TrIFNs. Through in-depth discussion of HM operator with TrIFN information, this study has enriched the theory of harmonic aggregation under intu- itionistic fuzzy environment. The desirable properties of the proposed operators have also been studied. The proposed operators are suitable tools, especially in the cases where the few outliers exist in the data set. An enterprise selection example is demonstrated to illustrate the utilization of the proposed operator for solving a MAGDM problem. Furthermore, comparison analysis is conducted to show the effectiveness of the proposed operators.

As stated earlier, in this work, the proposed operators are used in an enterprise selection problem; however, it can also be applied to any other areas of decision problems where uncertainty and hesitation are involved in the evaluation process and this will be our future research work.

\section{Acknowledgement}

The authors are very grateful to the Editors and the anonymous Reviewers for their constructive comments and suggestions. The first author gratefully acknowledges the financial support provided by the MHRD, Government of India.

\section{References}

1. Tian, Z.P., Wang, J., Wang, J.Q. and Chen, X.H. "Multicriteria decision-making approach based on gray linguistic weighted bonferroni mean operator", International Transactions in Operational Research (2015). DOI: 10.1111 /itor.12220.

2. Wan, S.P., Wang, F., Lin, L.L. and Dong, J.Y. "Some new generalized aggregation operators for triangular intuitionistic fuzzy numbers and application to multiattribute group decision making", Computers and Industrial Engineering, 93, pp. 286-301 (2016).

3. Ma, Y.X, Wang, J., Wang, J.Q. and Chen, X.H. “ Two-tuple linguistic aggregation operators based on subjective sensation and objective numerical scales for multi-criteria group decision-making problems", Scientia Iranica, 23(3), pp. 1399-1417 (2016).

4. Cho, S.B. "Fuzzy aggregation of modular neural networks with ordered weighted averaging operators", International Journal of Approximate Reasoning, 13(4), pp. 359-375 (1995).

5. Yager, R.R. and Filev, D. "Fuzzy logic controllers with flexible structures", In Proceedings of the 2nd International Conference on Fuzzy Sets and Neural Networks, lizuka, pp. 317-320 (1992).

6. Yager, R.R. "On ordered weighted averaging aggregation operators in multicriteria decision making", IEEE 
Transactions on Systems, Man and Cybernetics, 18, pp. 183-190 (1988).

7. Xu, Z. and Da, Q. "The ordered weighted geometric averaging operators", International Journal of Intelligent Systems, 17(7), pp. 709-716 (2002).

8. Chen, H., Liu, C. and Sheng, Z. "Induced ordered weighted harmonic averaging (IOWHA) operator and its application to combination forecasting method", Chinese Journal of Management Science, 12(5), pp. 35-40 (2004).

9. Ma, Y.X., Wang, J.Q., Wang, J. and Wu, X.H. "An interval neutrosophic linguistic multi-criteria group decision-making method and its application in selecting medical treatment options", Neural Computing and Applications, 28(9), pp. 2745-2765 (2017). DOI: $10.1007 / \mathrm{s} 0052101622031$

10. Zadeh, L.A. "Fuzzy sets", Information and Control, 8(3), pp. 338-353 (1965).

11. Atanassov, K.T. "Intuitionistic fuzzy sets", Fuzzy Sets and Systems, 20(1), pp. 87-96 (1986).

12. Yu, S.M., Zhou, H., Chen, X.H. and Wang, J.Q. "A multi-criteria decision-making method based on Heronian mean operators under a linguistic hesitant fuzzy environment", Asia-Pacific Journal of Operational Research, 32(5), pp. 1-35 (2015).

13. Wang, X. and Fan, Z. "Fuzzy ordered weighted averaging (FOWA) operator and its application", Fuzzy Systems and Mathematics, 17(4), pp. 67-72 (2003).

14. Xu, Z. "Fuzzy harmonic mean operators", International Journal of Intelligent Systems, 24(2), pp. 152172 (2009).

15. Yager, R.R. "The power average operator", IEEE Transactions on Systems, Man and Cybernetics, Part A: Systems and Humans, 31(6), pp. 724-731 (2001).

16. Choquet, G. "Theory of capacities", In Annales de Linstitut Fourier, 5, pp. 131-295 (1954).

17. Yager, R.R. "On generalized Bonferroni mean operators for multi-criteria aggregation", International Journal of Approximate Reasoning, 50(8), pp. 12791286 (2009).

18. Das, S., Dutta, B. and Guha, D. "Weight computation of criteria in a decision-making problem by knowledge measure with intuitionistic fuzzy set and intervalvalued intuitionistic fuzzy set", Soft Computing, 20(9), pp. 3421-3442 (2016). DOI: 10.1007/s00500-015-1813-3

19. Das, S., Guha, D. and Dutta, B. "Medical diagnosis with the aid of using fuzzy logic and intuitionistic fuzzy logic", Applied Intelligence, 45(3), pp. 850-867 (2016). DOI 10.1007/s10489-016-0792-0

20. Wan, S.P. and Li, D.F. "Atanassov's intuitionistic fuzzy programming method for heterogeneous multiattribute group decision making with Atanassov's intuitionistic fuzzy truth degrees", IEEE Transactions on Fuzzy Systems, 22(2), pp. 300-312 (2014).
21. Wan, S.P., Wang, F. and Dong, J.Y. "A novel group decision making method with intuitionistic fuzzy preference relations for RFID technology selection", Applied Soft Computing, 38, pp. 405-422 (2016).

22. Wan, S.P., Wang, F. and Dong, J.Y. "A novel risk attitudinal ranking method for intuitionistic fuzzy values and application to MADM", Applied Soft Computing, 40, pp. 98-112 (2016).

23. Wang, J.Q., Nie, R.R., Zhang, H.Y. and Chen, X.H. "Intuitionistic fuzzy multi-criteria decision-making method based on evidential reasoning", Applied Soft Computing, 13(4), pp. 1823-1831 (2013).

24. Xu, G.L., Wan, S.P., Wang, F., Dong, J.Y. and Zeng, Y.F. "Mathematical programming methods for consistency and consensus in group decision making with intuitionistic fuzzy preference relations", KnowledgeBased Systems, 98, pp. 30-43 (2016).

25. Wan, S.P. and Li, D.F. "Fuzzy LINMAP approach to heterogeneous MADM considering comparisons of alternatives with hesitation degrees", Omega, 41(6), pp. 925-940 (2013).

26. Wan, S.P. and Dong, J.Y. "Interval-valued intuitionistic fuzzy mathematical programming method for hybrid multi-criteria group decision making with intervalvalued intuitionistic fuzzy truth degrees", Information Fusion, 26, pp. 49-65 (2015).

27. Wan, S.P. and Li, D.F. "Fuzzy mathematical programming approach to heterogeneous multiattribute decision-making with interval-valued intuitionistic fuzzy truth degrees", Information Sciences, 325, pp. 484-503 (2015).

28. Wan, S.P., Wang, F., Lin, L.L. and Dong, J.Y. "An intuitionistic fuzzy linear programming method for logistics outsourcing provider selection", KnowledgeBased Systems, 82, pp. 80-94 (2015).

29. Xu, Z. "Intuitionistic fuzzy aggregation operators", IEEE Transactions on Fuzzy Systems, 15(6), pp. 11791187 (2007).

30. Xu, Z. and Yager, R.R. "Intuitionistic fuzzy Bonferroni means", IEEE Transactions on Systems, Man, and Cybernetics, Part B: Cybernetics, 41(2), pp. 568-578 (2011).

31. Das, S. and Guha, D. "A centroid-based ranking method of trapezoidal intuitionistic fuzzy numbers and its application to MCDM problems", Fuzzy Information and Engineering, 8, pp. 41-74 (2016).

32. Dong, J., Yang, D. and Wan, S.P. "Trapezoidal intuitionistic fuzzy prioritized aggregation operators and application to multi-attribute decision making", Iranian Journal of Fuzzy Systems, 12(4), pp. 1-32 (2015).

33. Wan, S.P. and Dong, J.Y. "Method of trapezoidal intuitionistic fuzzy number for multi-attribute group decision", Control Decision, 25(5), pp. 773-776 (2010).

34. Wan, S.P. and Dong, J.Y. "Multi-attribute group decision making with trapezoidal intuitionistic fuzzy numbers and application to stock selection", Informatica, 25(4), pp. 663-697 (2014). 
35. Wan, S.P. and Zhang, X. "Method based on weighted possibility mean for solving matrix games with payoffs of intuitionistic trapezoidal fuzzy numbers", Control and Decision, 27, pp. 1121-1126 (2012).

36. Wan, S.P., Zhang, X. and Li, D. "Possibility meanvariance based method for intuitionistic trapezoidal fuzzy group decision making", Journal of System and Engineering, 27, pp. 782-788 (2012).

37. Wang, C.H. and Wang, J.Q. "A multi-criteria decisionmaking method based on triangular intuitionistic fuzzy preference information", Intelligent Automation and Soft Computing, 22(3), pp. 473-482 (2016). DOI: 10.1080/10798587.2015.1095418

38. Das, S. and Guha, D. "Power harmonic aggregation operator with trapezoidal intuitionistic fuzzy numbers for solving magdm problems", Iranian Journal of Fuzzy Systems, 12, pp. 41-74 (2015).

39. Dong, J.Y. and Wan, S.P "A new method for multiattribute group decision making with triangular intuitionistic fuzzy numbers", Kybernetes, 45(1), pp. 158180 (2016).

40. Dong, J.Y. and Wan, S.P. "A new method for prioritized multi-criteria group decision making with triangular intuitionistic fuzzy numbers", Journal of Intelligent and Fuzzy Systems, 30(3), pp. 1719-1733 (2016).

41. Wan, S.P. "Multi-attribute decision making method based on possibility variance coefficient of triangular intuitionistic fuzzy numbers", International Journal of Uncertainty, Fuzziness and Knowledge-Based Systems, 21(02), pp. 223-243 (2013).

42. Wan, S.P. and Dong, J.Y. "Possibility method for triangular intuitionistic fuzzy multi-attribute group decision making with incomplete weight information", International Journal of Computational Intelligence Systems, 7(1), pp. 65-79 (2014).

43. Wan, S.P., Li, D.F. and Rui, Z.F. "Possibility mean, variance and covariance of triangular intuitionistic fuzzy numbers", Journal of Intelligent and Fuzzy Systems, 24(4), pp. 847-858 (2013).

44. Wan, S.P. and Li, D.F. "Possibility mean and variance based method for multi-attribute decision making with triangular intuitionistic fuzzy numbers", Journal of Intelligent and Fuzzy Systems, 24(4), pp. 743-754 (2013).

45. Wan, S.P., Wang, Q.Y. and Dong, J.Y. "The extended VIKOR method for multi-attribute group decision making with triangular intuitionistic fuzzy numbers", Knowledge-Based Systems, 52, pp. 65-77 (2013).

46. Wan, S.P. and Xu, J. "A method for multiattribute group decision making with triangular intuitionistic fuzzy numbers and application to trustworthy service selection", Scientia Iranica, Transactions on Industrial Engineering (Accepted).

47. Wang, J. and Zhong, Z. "Aggregation operators on intuitionistic trapezoidal fuzzy number and its application to multi-criteria decision making problems", Jour- nal of Systems Engineering and Electronics, 20(2), pp. 321-326 (2009).

48. Wan, S.P. and Dong, J.Y. "Multiattribute decision making based on triangular intuitionistic fuzzy numbers Choquet integral operator", Chinese Journal of Management Science, 22, pp. 121-129 (2014).

49. Wan, S.P., Lin, L.L. and Dong, J.Y. "MAGDM based on triangular atanassovs intuitionistic fuzzy information aggregation", Neural Computing and Applications, 27, pp. 1-16 (2016).

50. Dong, J.Y., Lin, L.L., Wang, F. and Wan, S.P. "Generalized Choquet integral operator of triangular Atanassov's intuitionistic fuzzy numbers and application to multiattribute group decision making", International Journal of Uncertainty, Fuzziness and Knowledge-Based Systems, 24, pp. 647-684 (2016).

51. Wan, S.P. and Zhu, Y.J. "Triangular intuitionistic fuzzy triple Bonferroni harmonic mean operators and application to multiattribute group decision making", Iranian Journal of Fuzzy Systems, 13(5), pp. 117-145 (2016).

52. Wu, J. and Cao, Q.W. "Same families of geometric aggregation operators with intuitionistic trapezoidal fuzzy numbers", Applied Mathematical Modelling, 37(1), pp. 318-327 (2013).

53. Liu, P. and Jin, F. "The trapezoid fuzzy linguistic Bonferroni mean operators and their application to multiple attribute decision making", Scientia Iranica, 19(6), pp. 1947-1959 (2012).

54. Wan, S.P. "Power average operators of trapezoidal intuitionistic fuzzy numbers and application to multiattribute group decision making", Applied Mathematical Modelling, 37(6), pp. 4112-4126 (2013).

55. Wan, S.P. and Dong, J.Y. "Power geometric operators of trapezoidal intuitionistic fuzzy numbers and application to multi-attribute group decision making", Applied Soft Computing, 29, pp. 153-168 (2015).

56. Wan, S.P. and Yi, Z. "Power average of trapezoidal intuitionistic fuzzy numbers using strict tnorms and t-conorms", IEEE Transaction on Fuzzy Systems, 24(5), pp. 1035-1047 (2016). DOI:10.1109/TFUZZ.2015.2501408

57. Dong, J.Y. and Wan, S.P. "Interval-valued trapezoidal intuitionistic fuzzy generalized aggregation operators and application to multi-attribute group decision making", Scientia Iranica, Transactions on Industrial Engineering, 22(6), pp. 2702-2715 (2015).

58. Wan, S.P. "Multi-attribute decision making method based on interval-valued intuitionistic trapezoidal fuzzy number", Control and Decision, 26(6), pp. 857861 (2011).

59. Wan, S.P. "Method based on fractional programming for interval-valued intuitionistic trapezoidal fuzzy number multi-attribute decision making", Control and Decision, 27(3), pp. 455-458 (2012).

60. Das, S. and Guha, D. "Ranking of intuitionistic fuzzy number by centroid point", Journal of Industrial and Intelligent Information, 1(2), pp. 107-110 (2013). 
61. Matuszak, A. "Differences-arithmetic-geometricharmonic-means", The Economist at Large (2010). http: econo- mistatlarge.com/finance/applied-finance/ differences-arithmetic-geometric-harmonic-means

62. Chen, S.M. and Tan, J.M. "Handling multicriteria fuzzy decision-making problems based on vague set theory", Fuzzy Sets and Systems, 67(2), pp. 163-172 (1994).

\section{Biographies}

Satyajit Das received his MSc degree in Mathematics with Computer Applications from NIT, Durgapur, India in 2012. He has received his $\mathrm{PhD}$ degree in Mathematics from IIT Patna, India in 2017. His current research area includes: fuzzy optimization, multi-criteria decision making, and intuitionistic fuzzy set theory.

Debashree Guha received her PhD degree in Mathematics from IIT Kharagpur, India in 2011. Presently, she is an Assistant Professor at the Department of Mathematics in IIT, Patna, India. Her current research interest includes: multi-attribute decision making, fuzzy mathematical programming, aggregation operators and fuzzy logic. Her research results have been published in the journals: IEEE Transactions on Fuzzy Systems, IEEE Transactions on Systems, Man and Cybernetics: Systems, Applied Soft Computing, Soft Computing, among others. 